\title{
Consolidating behavioral and neurophysiologic findings to explain the influence of contextual interference during motor sequence learning
}

\author{
David Wright $^{1} \cdot$ Willem Verwey $^{2} \cdot$ John Buchanen $^{1} \cdot$ Jing Chen $^{1} \cdot$ Joohyun Rhee $^{1}$. \\ Maarten Immink ${ }^{3}$
}

Published online: 18 June 2015

(C) Psychonomic Society, Inc. 2015

\begin{abstract}
Motor sequence learning under high levels of contextual interference (CI) disrupts initial performance but supports delayed test and transfer performance when compared to learning under low CI. Integrating findings from early behavioral work and more recent experimental efforts that incorporated neurophysiologic measures led to a novel account of the role of CI during motor sequence learning. This account focuses on important contributions from two neural regions-the dorsal premotor area and the SMA complex-that are recruited earlier and more extensively during the planning of a motor sequence in a high CI context. It is proposed that activation of these regions is critical to early adaptation of sequence structure amenable to long-term storage. Moreover, greater CI enhances access to newly acquired motor sequence knowledge through (1) the emergence of temporary functional connectivity between neural sites previously described as crucial to successful longterm performance of sequential behaviors, and (2) heightened excitability of M1-a key constituent of the temporary coupled neural circuits, and the primary candidate for storage of motor memory.
\end{abstract}

David Wright

d-wright@hlkn.tamu.edu

1 Department of Health and Kinesiology, Texas A\&M University, 4243 TAMU, College Station, TX 77843-4243, USA

2 Department of Cognitive Psychology and Ergonomics, University of Twente, Twente, The Netherlands

3 Human Movement, School of Health Sciences, University of South Australia, Adelaide, Australia
Keywords Contextual interference $\cdot$ Motor sequence learning $\cdot$ Practice schedule $\cdot$ Motor learning

\section{Introduction}

Determining how motor skills are acquired and detailing the associated neuroplasticity resulting from extended practice or experience has garnered extensive experimental rigor in recent years (Dayan \& Cohen, 2011; Doyon et al., 2009; Karni et al., 1998). To fully appreciate the learning process it is crucial to consider not only practice extent but also the type and structure of practice as ways to enhance motor skill proficiency. For example, there is growing evidence that non-physical forms of practice, such as observation, are sufficient to support successful retention and transfer (Black, Wright, Magnuson, \& Brueckner, 2005; Buchanan \& Wright, 2011; Buchanan, Zihlman, Ryu, \& Wright, 2007; Carroll \& Bandura, 1982, 1990; Scully \& Newell, 1985). Moreover, a variety of practice schedules have been associated with substantial benefits for motor learning. One in particular that has attracted considerable attention over the past 30 years, studied under the rubric of the contextual interference (CI) effect, focuses on best practice for improving the acquisition of multiple, related motor skills (Brady, 1998, 2004; Magill \& Hall, 1990; Shea \& Morgan, 1979).

During experiments addressing the impact of CI for motor learning, greater CI is often engineered by arranging training such that the learner executes multiple motor tasks in a random format. Random practice, as it is called, creates relatively high interference throughout training because of the rapid changes in task demands across trials. Conversely, blocked practice creates less interference because it entails executing the same motor task repeatedly prior to the introduction of alternative 
to-be-learned motor tasks that will be practiced later in training. ${ }^{1}$ Of greatest interest is the counterintuitive finding that experiencing random as opposed to block practice, i.e., experiencing greater rather than less interference, disrupts initial performance but supports superior delayed retention and transfer (see Fig. 1). For motor learning this finding is robust having been observed for a wide variety of laboratory tasks (Pauwels, Swinnen, \& Beets, 2014; Shea \& Morgan, 1979; Wright, Magnuson, \& Black, 2005) and in many applied situations (Goode \& Magill, 1986; Ollis, Button, \& Fairweather, 2005; Smith \& Davies, 1995). The efficacy of a high CI practice schedule for improved retention and transfer benefit has been demonstrated for a variety of subject populations (Del Rey, 1982; Porretta \& Obrien, 1991), and has been used in the clinical domain with some success (Adams \& Page, 2000; Knock, Ballard, Robin, \& Schmidt, 2000; Wambaugh, Nessler, Wright, \& Mauszycki, 2014).

The veracity of the CI effect is rarely questioned (although see, Russell \& Newell, 2007) as the general effect has been replicated and has intuitive practical appeal (Brady, 2004, 2008; Magill \& Hall, 1990). Nonetheless, the underlying theoretical basis for its emergence is poorly understood and there is a dearth of focused effort to resolve this shortcoming. For some time now there have been two general descriptions, the forgetting-reconstruction (Lee \& Magill, 1983, 1985) and the elaboration (Shea, Hunt, \& Zimny, 1985; Shea \& Zimny, 1983) accounts, rooted in the information processing tradition and developed solely on the basis of behavioral findings.

The forgetting-reconstruction position, hereafter referred to as the reconstruction account, focuses primarily on unspecified reconstructive processes that occur more extensively as part of motor sequence preparation in high CI training environments (Lee \& Magill, 1983, 1985). Essentially, Lee and colleagues claim that, during random practice, the learner must contend with intervening responses between successive attempts on any single motor task. Such intervening activity is assumed to instigate significant forgetting of task-specific information between consecutive trials necessitating the (re)construction of an action plan for each response. In doing so, the random practice participant becomes relatively more adept than their blocked practice counterpart at "the process of developing and implementing an action plan" (p. 19, Lee \& Magill, 1985). The focus of this account then is on the improvement in effectively manufacturing the appropriate

\footnotetext{
${ }^{1}$ While random and blocked practice formats are the most frequent scheduling procedures used to change the amount of contextual interference created in a practice bout, other practice formats have been used. For example a serial practice has been adopted in a number of studies (e.g., Lee \& Magill, 1983).
}

plan for each upcoming response as a result of being exposed to heightened interference in practice.

In keeping with the reconstruction position, the elaboration account also posits that differential processing is mandated by different practice schedules during practice (Shea et al., 1985; Shea \& Zimny, 1983). Specifically, Shea and Zimny referenced two qualitatively unique categories of information processing activity that the learner can entertain during practice. Intra-task processing consists of task analyses that exclude reference to information related to other motor tasks currently being acquired and/or other related knowledge presently available to the learner. Shea and Zimny argue that intra-task processing is the primary mode of operation during blocked practice. In contrast, inter-task processing involves the extraction of relationships or associations between the tasks currently being acquired as well as with pertinent long-term knowledge. Engaging inter-task processing requires that information about multiple motor sequences is simultaneously present in working memory, which is more likely during random practice. The elaboration account, however, goes beyond suggesting that the processing engaged during training is practice schedule dependent, suggesting that, as a result of these unique processing activities, both the knowledge of the motor task and the memory architecture in which it is distributed is fundamentally different following random and blocked training (Shea \& Zimny, 1983).

\section{Scope and limitations of the review}

Prior to delving into some of the latest findings central to offering a more contemporary account of how CI impacts motor learning it is important to recognize that, given the abundance of experimental effort addressing this practice phenomenon, it is inevitable that some work is highlighted at the expense of other studies (Magill \& Hall, 1990). As a consequence of emphasizing certain experimental work, boundaries emerge that potentially limit the scope of any review. This is certainly true for the present endeavor. For example, while there are some examples of the effectiveness of increased CI during training outside the traditional purview of motor skill acquisition (Carlson, Khoo, Yaure, \& Schneider, 1990; Carlson \& Yaure, 1990), it is hard to ignore that the vast majority of studies addressing CI have focused on skill acquisition in the motor domain. Motor learning is intentionally the domain of interest in the present work.

As noted in the previous section, the type of motor skills that have been the subject of many of the experimental efforts to evaluate the impact of $\mathrm{CI}$ for motor learning is quite varied (Pauwels et al., 2014; Shea \& Morgan, 1979; Wright et al., 2005). Nonetheless, most of the basic and applied work has involved motor sequence learning. This class of motor skill, in conjunction with the motor adaptation skill, was recently 


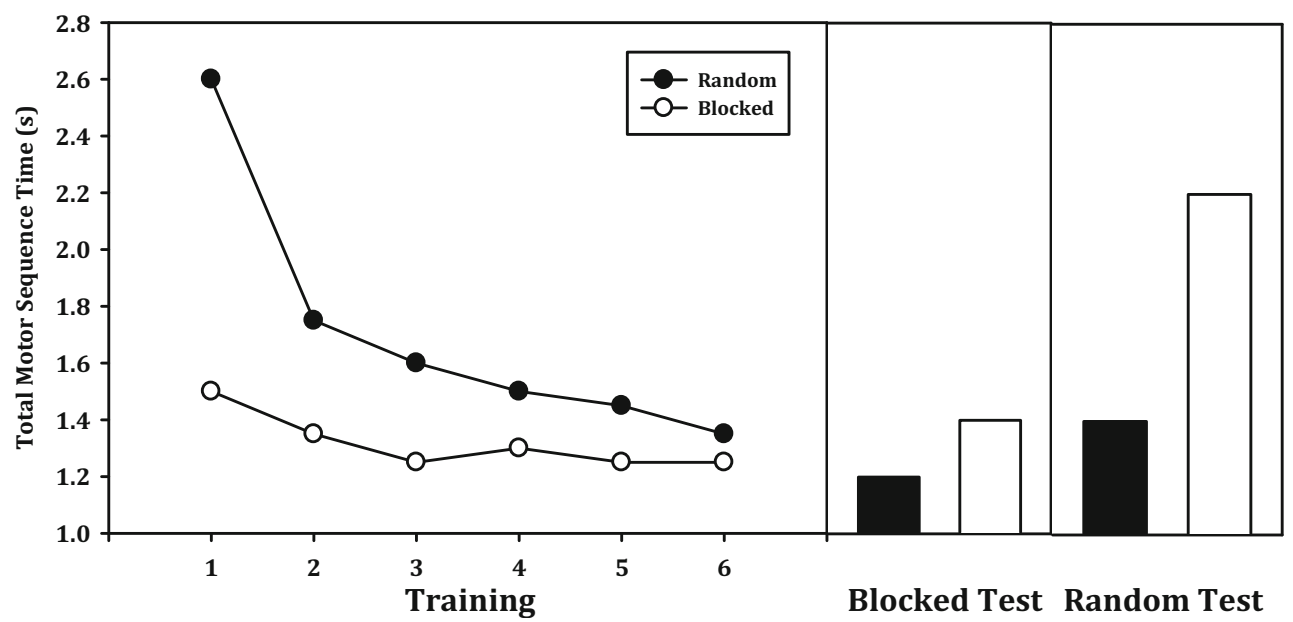

Fig. 1 The contextual interference (CI) effect is commonly characterized by superior performance in the training (acquisition) phase by individuals assigned to a blocked practice, assumed to induce a low level of CI (white symbols) compared to participants exposed to random practice (black symbols), a schedule involving relatively greater CI. Of greatest interest

recognized as a critical category of skilled motor behavior that needs to be considered when describing the process of motor learning (Doyon et al., 2009). Indeed, for the purpose of the present work it is assumed that a wide range of tasks from variations of the discrete sequence production task (Abrahamse, Ruitenberg, de Kleine, \& Verwey, 2013), to basketball (Porter \& Magill, 2010), surgical (Dubrowski, Backstein, Abughaduma, Leidl, \& Carnahan, 2005) or speech skills (Wambaugh et al., 2014) can be characterized as motor sequence tasks. It is further assumed that the present account of how CI impacts learning extends across the entire range of motor sequence tasks.

Despite the intent to provide a contemporary account of how increased CI facilitates motor sequence learning, it would be misleading to ignore the fact that significant emphasis throughout this work is on experimental evidence from studies that used variations of the discrete sequence production task (DSPT) (Abrahamse et al., 2013). This is a consequence of the fact that almost all of the available studies designed to expose information processing differences during blocked and random practice utilize a variation of this task type. For example, early experimental work evaluated how random and blocked practice formats facilitate the production of DSPTs that varied across relative and absolute time dimensions (Lee, Wulf, \& Schmidt, 1992; Magnuson \& Wright, 2004; Wulf \& Lee, 1993) or how relative time emerged for a set of DSPTs when practicing within a high or low CI format (Immink \& Wright, 2001; Wright, Brueckner, Black, Magnuson, \& Immink, 2004) . More recent endeavors, that combine the traditional behavioral approach with neurophysiological assessment, have relied almost exclusively on the use of DSPTs that are executed with the correct ordinal structure as quickly as possible. It is likely that the nature of the DSPTs are simplified in is the counter-intuitive observation that achievement during delayed test phases (histograms on right panels) is better for those individuals that experienced greater CI during training. Note here that lower values for total motor sequence time (s) are better. Data adapted from Shea and Morgan (1979)

more recent work because the primary focus is on providing important novel insights into the neural underpinnings of learning that occurs within the confines of practice schedules inducing various levels of CI (Lin, Fisher, Winstein, Wu, \& Gordon, 2008; Lin, Winstein, Fisher, \& Wu, 2010; Wymbs \& Grafton, 2009).

Finally, given this review specifically targets motor sequence learning, it is essential to recognize that this form of learning can be accomplished via explicit or implicit means (Destrebecqz \& Cleeremans, 2001; Nissen \& Bullemer, 1987; Willingham, 1998). Indeed, there is growing evidence suggesting that explicit and implicit learning strategies adopted during motor learning are distinct and supported by unique neural circuits (Grafton, Hazeltine, \& Ivry, 1995; Hikosaka et al., 1999; Hikosaka, Nakamura, Sakai, \& Nakahara, 2002). We know of only a few studies that have specifically manipulated CI during implicit motor sequence learning either directly (Rendell, Masters, \& Farrow, 2009; Sekiya, 2006; Song, Sharma, Buch, \& Cohen, 2012) or indirectly (Pauwels et al., 2014). As such, it is acknowledged that the relevance of this work pertains to the influence of CI during motor sequence learning that occurs via the explicit system.

\section{Increasing contextual interference during practice increases the attention demands associated with planning an upcoming motor sequence}

As alluded to earlier, the elaboration and reconstruction positions have been viewed traditionally as competing theoretical accounts of the CI effect (Lin et al., 2008; Young, Cohen, \& Husak, 1993). Others have argued that features of the two positions merely target different contributions to learning that 
can be garnered through exposure to high CI practice (Immink \& Wright, 2001; Wright et al., 2004). Despite this debate, it is generally accepted that both perspectives place a heavy emphasis on unique trial-to-trial cognitive operations (i.e., reconstruction, inter-task evaluation) that are encouraged when greater $\mathrm{CI}$ is present during practice. A reasonable starting place to begin this review then is to examine a common prediction emerging from the elaboration and reconstruction accounts, that is, encountering greater $\mathrm{CI}$ in practice is "more effortful or demanding" due to the need to engage a broader set of cognitive operations needed to execute an action.

The veracity of this prediction was addressed directly by $\mathrm{Li}$ and Wright (2000), who hypothesized that additional cognitive activity in a high CI practice condition should be associated with a concomitant increase in attention demand compared to blocked practice. Li and Wright used a dual-task paradigm in which individuals experienced either random or blocked practice of three separate motor sequences while performing a secondary choice reaction time (CRT) task that involved tone discrimination. Across practice, the secondary task was presented at two distinct temporal intervals within a single trial. Specifically, the secondary CRT task was periodically presented shortly after the participant was informed about the motor sequence to be performed on the next trial, presumably while the performer was assumed to be constructing an action plan (Lee \& Magill, 1985). Alternatively, the CRT task was administered in the inter-trial interval, which was assumed to capture the demands of comparative operations that are the hallmark of inter-task processing assumed to occur in high CI practice formats (Wright, 1991).

The data revealed greater attention demand during random compared to blocked practice as evidenced by a significantly larger increase in secondary CRTs during practice. The difference in attention demand between the practice formats was most pronounced during the time interval that reconstructive activity was thought to occur ( $20 \%$ increase in secondary task CRT) compared to the inter-trial interval during which the comparative process including inter-task evaluation should be undertaken $(\sim 7 \%$ increase in secondary task CRT) (Li \& Wright, 2000). This finding was congruent with the core claim of both theoretical positions that increasing $\mathrm{CI}$ in training encourages more active attention-demanding motor planning on the part of the learner, which in turn impacts negatively on the rate of performance improvement during training (also see, Immink \& Wright, 1998).

\section{Accounting for increased attention demand during a high CI practice environment}

An important next step then is to address the more elusive issue of detailing the specific nature of the reported extra attention demand during random practice (Li \& Wright, 2000).
A few early efforts to delineate the underlying reasons for the larger demand during random practice used only a behavioral approach but more recent ventures have incorporated neurophysiologic methods. Findings from recent studies have uncovered differences in the nature of the cognitive processes that occur during preparation of a motor sequence in a low and high CI training environments. Some of these differences have critical relevance for determining delayed performance capacity. Relevant data from early behavioral efforts as well as more recent efforts delineating the unique neural recruitment patterns adopted as a consequence of different practice schedules are discussed in the next section.

\section{More extensive motor programming of a motor sequence occurs during practice that induces greater contextual interference}

The initial behavioral work designed to uncover the locus of the larger attention demand during random as opposed to blocked practice adopted the traditional sequential stage model as a theoretical backdrop (Immink \& Wright, 2001; Sternberg, 1969). In particular, the motor programming process, which is considered part of response preparation responsible for organizing parameters of a selected action was the target of this line of investigation (Klapp, 1995; Sternberg, 1969). The motor programming process was considered a reasonable starting point to more precisely identify the preparatory processes differentially impacted by random and blocked practice for a couple of different reasons, detailed by Immink and Wright (2001). First, the description of "reconstruction" offered by Lee and Magill $(1983,1985)$ is consistent with "late" response preparation processes as opposed to relatively earlier perceptual-type processes that must also be conducted prior to response execution. Second, motor programming has been described as a "function of complexity or duration of the response because of a difference in the time required to reconstruct a response representation from a code in LTM" (Klapp, 1996, p14). This description appeared, at least at the outset, to be within the spirit of Lee and Magill's theoretical position.

To evaluate the influence of $\mathrm{CI}$ on motor programming during response preparation, Immink and Wright (2001) used a more detailed description of motor programming forwarded by Klapp $(1995,1996)$. This account identifies two distinct motor programming processes that are independently responsible for (1) retrieving the action's building blocks, often termed "motor chunks," called the INT process (Verwey, 1999), and (2) organizing the motor chunks into the correct serial order for output, referred to as the SEQ process. Importantly, Klapp proposed that the INT process, retrieval of the necessary motor chunks, can be preprogrammed while the SEQ process, planning the sequential order of the retrieved motor chunks, must occur after presentation of the imperative signal. Using the selfselect paradigm (see Fig. 2a) Immink and Wright (2001) had 
a

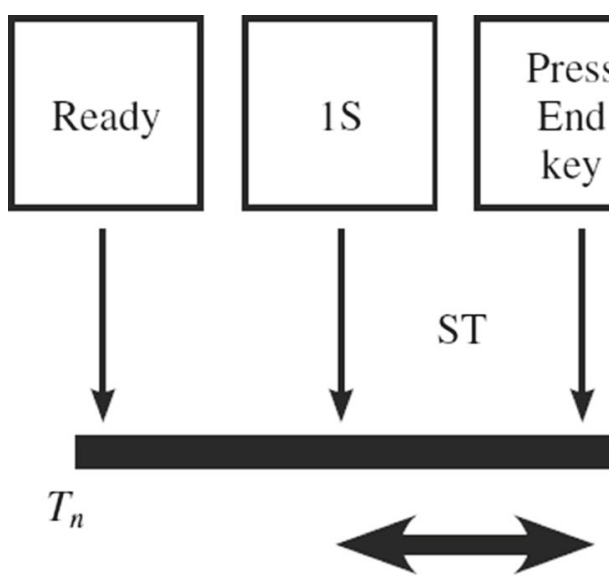

INT

Process

b

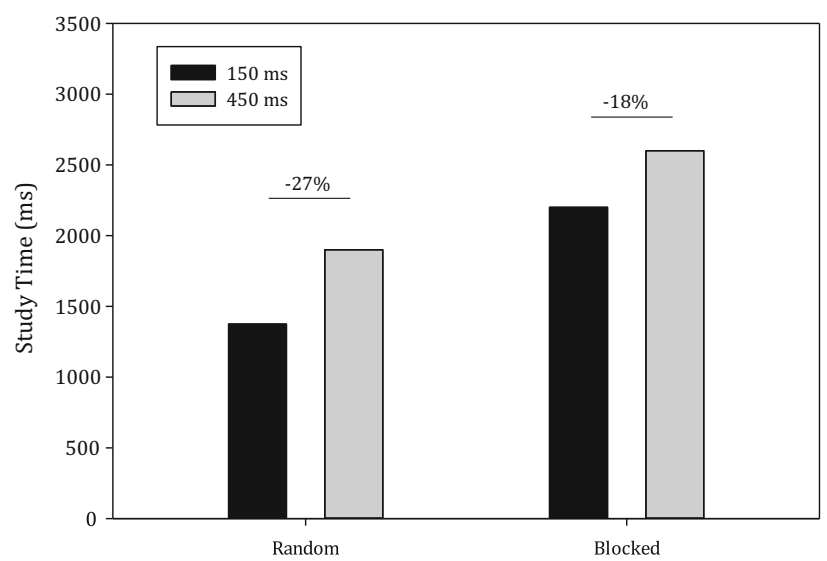

Fig. 2 a The self-select paradigm can be incorporated with a CI study in which multiple tasks are being practiced. On any single trial a "ready" signal presented on a computer monitor starts a trial. The participant is then provided a precue on the computer monitor about the motor sequence that must be prepared to be executed for that trial. In the example provided from Immink and Wright (2001) this is a single key press of short duration (indicated by $1 S$ ). Study time (ST) is defined operationally as the interval between the presentation of the precue and the participant pressing the "End" key to indicate they have readied a response. This interval is assumed to capture the temporal demands of the INT motor programming process. After the "END" key is pressed a variable fore period occurs before a "GO" signal is presented. Reaction time (RT) is defined operationally as the interval between the presentation of the "GO" signal and the depression of the first response key in the required motor sequence. The RT interval captures the temporal demands

individuals practice short $(150 \mathrm{~ms})$ and long $(450 \mathrm{~ms})$ duration key presses as single element responses or as part of multipleelement sequences in either blocked or random practice schedules. Retrieving a $150 \mathrm{~ms}$ rather than $450 \mathrm{~ms}$ single element response was assumed to impart less demand on the INT process (i.e., less complex motor chunk, see Klapp, 1995; 1996), which should be reflected in a shorter study time in the selfselect paradigm because INT is pre-programmed (see Fig. 2a).

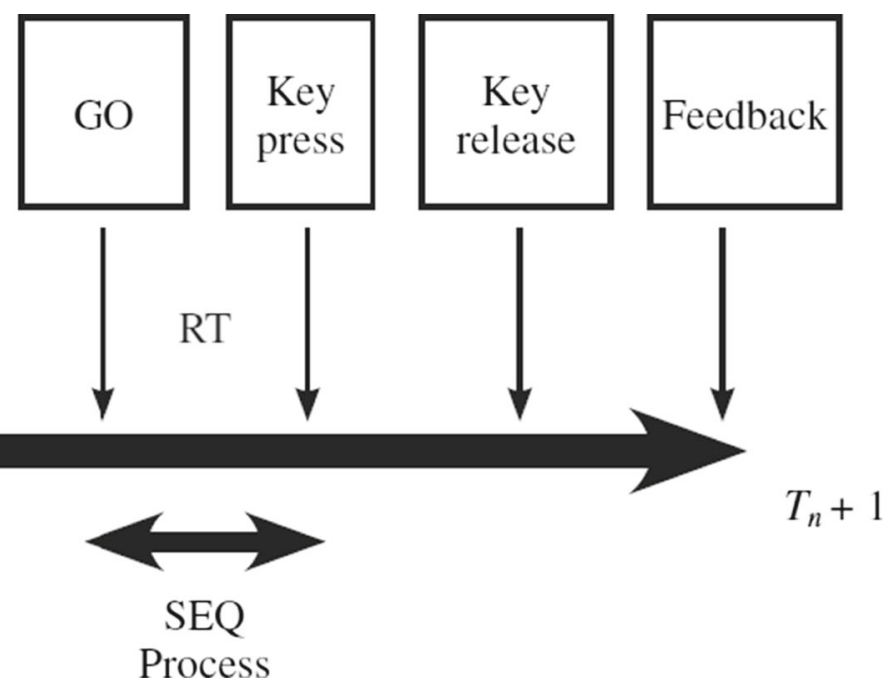

C

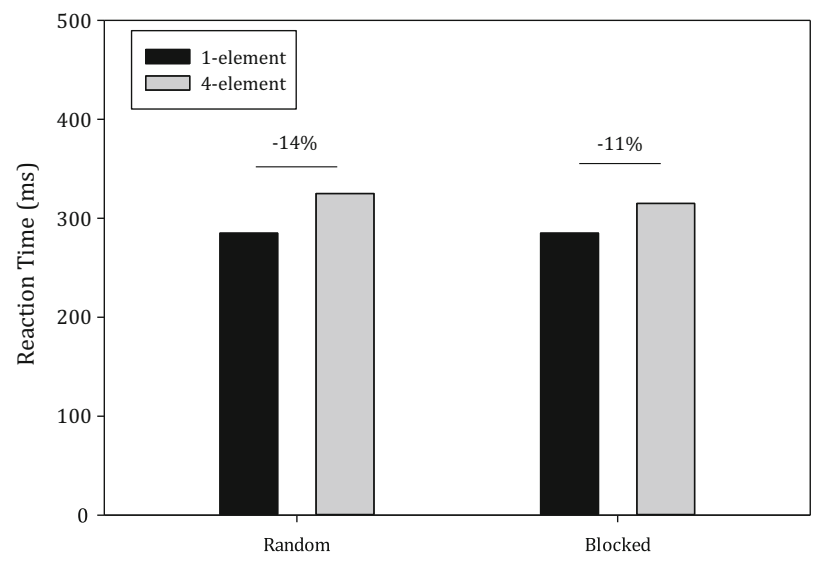

of the motor programming process labeled SEQ (see Klapp, 1995). b During the retention test, individuals assigned to random practice revealed lower study time when preparing the short and long duration single element responses compared to blocked practice indicating a more efficient employment of the INT (selecting and retrieving motor chunks) process. Percentages indicate the savings in study time when planning the easier response (i.e., short duration response in this case). (Data adapted from Immink \& Wright, 2001, Experiment 1). c During the retention test, $\mathrm{RT}$ is longer when preparing multiple element sequences rather than for single-element responses. The difference in RT between single- and multiple-element responses is not dependent on practice structure. Percentages indicate the savings in RT when planning the easier response (i.e., single rather than multi-element response in this case). (Data adapted from Immink \& Wright, 2001, Experiment 2)

Conversely, the cost incurred for the SEQ process is reflected in reaction time within the self-select paradigm as this process is implemented post-imperative signal (see Fig. 2a). The burden on the SEQ process was expected to be greater when preparing a multi-element sequence compared to a single-element sequence (see Klapp, 1995, 1996). In random practice a lower study time was observed when preparing the short and long duration single element responses compared to 
blocked practice indicating a more efficient employment of the INT (selecting and retrieving motor chunks) process (see Fig. 2b). As expected, reaction time was longer when preparing multiple element sequences rather than for single-element response; however, the difference in reaction time between single- and multiple-element responses was not dependent on practice structure (see Fig. 2c). These data suggested that being exposed to greater $\mathrm{CI}$ in practice does not influence how the SEQ process is conducted.

The latter interpretation of the SEQ process was modified in a subsequent study where the amount of practice was increased (Wright et al., 2004). With more practice, greater CI not only supported the INT process benefit, but also a sizeable improvement in the SEQ process. The improvement in the SEQ process occurred because of a reorganization of the motor sequence such that it involved fewer motor chunks. Individuals exposed to blocked practice also improved sequence execution during training by reducing the number of motor chunks. However, after only a 24-h delay, blocked practice participants returned to preparing their responses using a larger number of motor chunks whereas the random practice participants continued to use the motor chunk organization established at the conclusion of practice.

The finding that a high CI practice environment supports the development and maintenance of motor chunks is important because establishing motor chunks has been described as a fundamental strategy used to facilitate continued improvement in the performance of complex serial behaviors and considered central to motor skill learning (Sakai, Kitaguchi, \& Hikosaka, 2003; Verwey \& Eikelboom, 2003). For example, contemporary theoretical accounts for motor sequence learning propose that early control of a motor sequence is at the individual element level, referred to as the reaction mode (Abrahamse et al., 2013). As practice progresses performance of the motor sequence is accomplished by adopting an associative or chunking mode of operation which takes advantage of the structure in the perceptual or execution aspects of the motor sequence respectively (see Fig. 3). One interpretation of the findings of Wright and colleagues (Immink \& Wright, 2001; Wright et al., 2004) is that both random and blocked practice encourages early adoption of the chunking mode but only individuals in random practice store the developed motor chunks such that they can be retrieved and used at a later time to support sequence execution. This storage may involve ongoing migration of sequence knowledge, that is, motor chunks, to alternative neural sites that have been associated with long-term storage of motor sequence knowledge (Penhune \& Steele, 2012).

Assuming that the SEQ process involves some form of retrieval process as part of selecting the retrieved motor chunks for execution in the appropriate serial order (see, Klapp, 1996; Magnuson, Wright, \& Verwey, 2004;
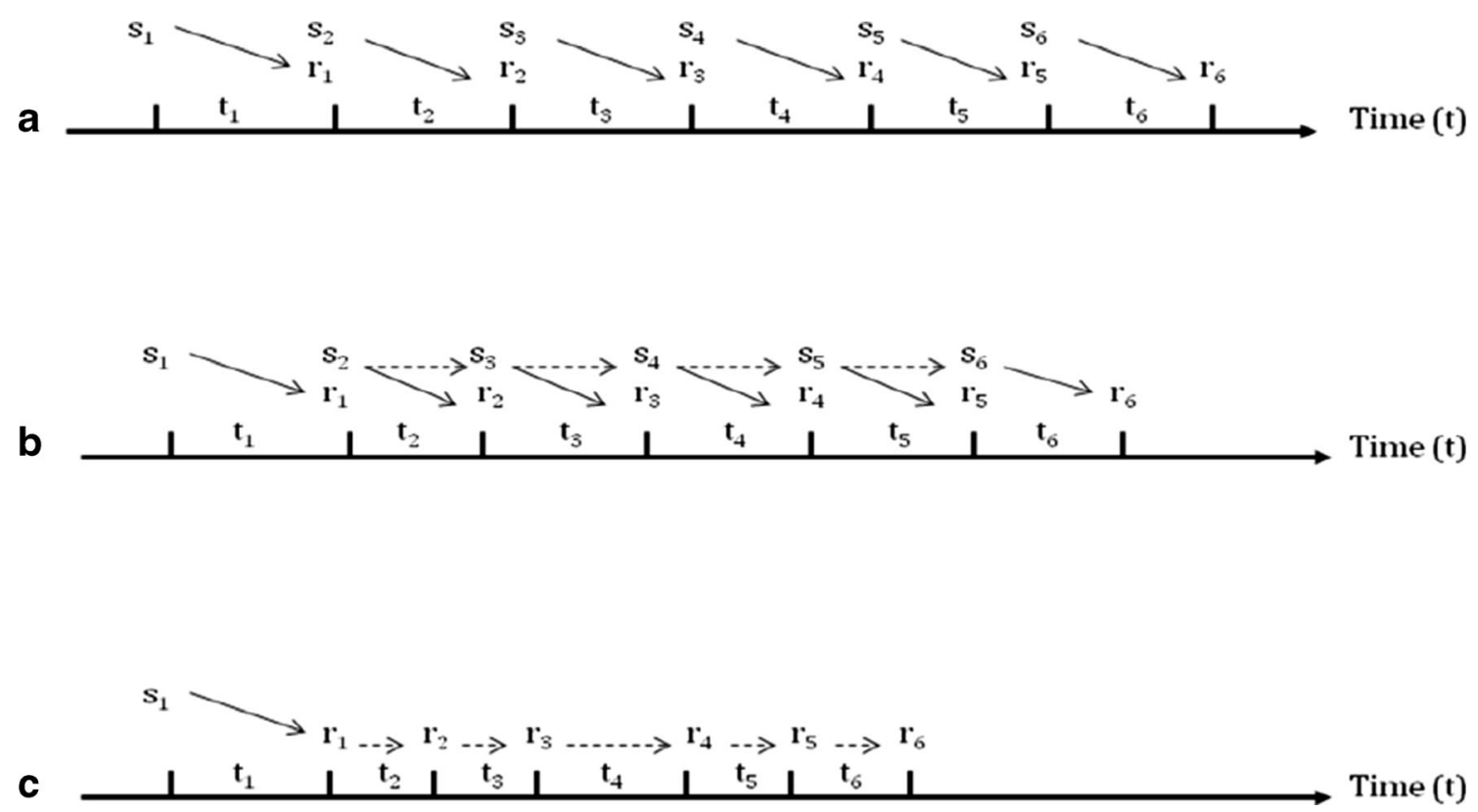

Fig. 3 The execution of a motor sequences can be occur via any one of three unique modes of control (Abrahamse et al., 2013). In the first case (a) each stimulus $\left(\mathrm{s}_{\mathrm{i}}\right)$ of the motor sequence is presented and the performer responds $\left(\mathrm{r}_{\mathrm{i}}\right)$ to this stimulus in time $\left(\mathrm{t}_{\mathrm{i}}\right)$ and this process is independent of responses to all other S-R pairs contained in the motor sequence. This is referred to as the reaction mode, which is used early in learning. With additional practice, the string of stimuli that designate a particular motor sequence become associated (i.e., form s-s relationships) such that stimulus $\mathrm{s}_{\mathrm{n}}$ can activate or prime stimulus $\mathrm{s}_{\mathrm{n}+1}$ thereby speeding up

the production of response $\mathrm{n}_{\mathrm{n}+1}$. In this case, further improvement in performance results from using the associative mode (b). Finally with extensive practice, participants can perform the entire motor sequence in the absence of all but the first stimulus. Moreover, the production of the motor sequence is parsed into functional units, called motor chunks, each of which appears to be recalled and executed in a single step. This is characteristic of the chunking mode and according to Abrahamse et al. is used when the learner is more skilled 
Sternberg, 1969) then it is not difficult to understand how consolidating the representations of a motor sequence as fewer motor chunks would facilitate planning. Yet, one might argue that, as this process unfolds, the resultant motor chunks, while smaller in number, are likely more elaborate and therefore should place a greater load on the INT process (i.e., retrieving individual motor chunks). If this is true, the improvement in programming a motor sequence, acquired during random practice, merely involves a re-distribution of the planning cost across different reconstructive processes. That is, costs are shifted from the SEQ to the INT process. From a functional standpoint one might question the utility of just re-diverting cognitive demands from one motor programming process to another. However, this strategy, if true, would still provide the performer a significant advantage because Klapp's (1995) model proposes that the INT, but not the SEQ process, can be preplanned, thus allowing the performer to complete more task organization in advance of an imperative signal. However, as it turns out, not only does random practice support the reduction of the temporal costs associated with the SEQ process by consolidating the sequential behavior into fewer motor chunks but this is done without a concomitant increase in the cost of conducting the INT process. This is revealed as a reduction in study time later in practice and during delayed tests. Thus, more extensive random practice facilitates (1) more efficient retrieval (i.e., INT process) of the pertinent motor chunks as well as (2) a more efficient organization of the motor chunks into the correct serial order prior to execution (i.e., the SEQ process).

Verification of the efficacy of random practice for improving the efficiency of motor programming during motor sequence learning was offered by Wright, Magnuson, and Black (2005) using a "precuing" protocol (Rosenbaum, 1980). In Wright et al. (2005), individuals practice two short key sequencing tasks that required a precise timing structure in either a random or blocked schedule. This was then followed by an assessment of the learner's capacity to either quickly compile or modify the learned procedural task's required timing structure. Within the precuing protocol, "compilation" of the motor sequence was examined by providing partial timing information in the form of a precue that was eventually followed by the remaining requisite timing information being part of the imperative signal. Thus, the performer had to complete the programming process by identifying the remaining information needed to completely plan the action prior to initiation. In contrast, "modification" of the motor sequence involved responding to imperative signals that followed a precue of a timing profile that had a high probability of occurrence ( $75 \%$ of trials) or imperative signals that were incongruent ( $25 \%$ of trials) with the precued timing information. In the latter case, programming of the action plan was assumed to require modification as a result of the information contained in the imperative signal. The most critical finding was further demonstration of more efficient motor programming following training that involved greater CI. This was revealed as lower RTs reflecting faster programming, while also displaying superior accuracy in response production, for the random as opposed to the blocked-trained individuals. This was especially pronounced when "modification" rather than "compilation" of timing was needed.

In summary, empirical evidence (Immink \& Wright, 1998; Wright et al., 2005), emanating from quite distinct behavioral paradigms (e.g., self-select, pre-cuing), point to a differential investment in the motor programming process during random and blocked practice (Lee \& Magill, 1983, 1985). Handling the rigors of programming motor sequences, when faced with greater CI, results in the adoption of the chunking mode which has been described as an important strategy for successful longterm performance of motor sequences (Abrahamse et al., 2013). Most importantly, the developed motor chunks are stored, thus available, for later use following random but not blocked practice. A greater commitment to the motor programming process during practice may account, at least in part, for some of the added attention demand associated with a high CI practice (Immink \& Wright, 1998; Li \& Wright, 2000).

The findings regarding motor programming and high CI practice formats does not, of course, preclude the possibility that other cognitive processes might also be improved from experiencing greater $\mathrm{CI}$ during training. There are a few studies available that indicated that increasing $\mathrm{CI}$ during practice might also help learning that is often consider separate from the realm of motor sequence learning such as problem solving (Carlson \& Yaure, 1990; de Croock, van Merrienboer, \& Paas, 1998). Indeed, there are data available that reveal a positive impact from random-type practice for perceptual (Fendrich, Healy, \& Bourne, 1991) and selection processes (Pashler \& Baylis, 1991; Wright, Magnuson, \& Verwey, 2003) conducted prior to motor programming. These findings need to be verified and examined in a more rigorous manner within the context of studies explicitly designed to examine $\mathrm{CI}$ and motor learning. Clearly this is one arena in which future behavioral research might offer further insight into the impact of this practice schedule phenomenon as well as make important theoretical contributions.

\section{A broader recruitment of sequence-specific neural regions occurs during a high contextual interference practice environment that tax more than just the motor programming process}

The previous section indicated that the extensiveness of sequence planning, especially the motor programming process, is dependent on the degree of $\mathrm{CI}$ induced during practice. It seems reasonable to assume then that the profile of neural recruitment during low and high CI practice formats should reflect, at a minimum, this differential planning demand. 
Moreover, it is also possible that careful examination of novel neurophysiologic data, collected in conjunction with behavioral work focused on CI during motor sequence learning, might provide additional insights into reasons for the additional attention costs associated with random as opposed to blocked practice (Immink \& Wright, 1998; Li \& Wright, 2000).

Initial neurophysiologic evidence was reported by Wymbs and Grafton (2009) from a study that involved executing multiple motor sequences in a random or blocked format using a go/no-go paradigm designed to separate preparatory (i.e., those of primary interest) and execution process during practice only (Cross, Schmitt, \& Grafton, 2007). As expected, the behavioral data revealed the expected effect of CI during acquisition and test (Shea \& Morgan, 1979). With respect to the neurophysiologic data random practice participants maintained a constant level of activation within some motor planning regions while increasing the activity in other regions that have been reported to be central to improvement in motor sequence performance across practice (Dayan \& Cohen, 2011; Doyon et al., 2009; Floyer-Lea \& Matthews, 2005; Hardwick, Rottschy, Miall, \& Eickhoff, 2013; Penhune \& Steele, 2012). Specifically, lateral (dorsal and ventral premotor) and medial (pre supplementary motor area (pSMA), supplementary motor area proper (SMA)) premotor areas exhibited a blood-oxygenated level dependent (BOLD) signal that was at least $50 \%$ greater later in practice than earlier, implying significant action preparation continued late into random training. The lateral premotor areas have been recognized for the development of associations between arbitrary sensory stimuli and actions and/or learning arbitrary response rules, which would certainly be an early challenge when learning a new set of motor sequences. The medial premotor areas, including pSMA and SMA proper, have been associated with selecting, retrieving, and organizing subsets or whole movement sequences at different points of skill acquisition (Gerloff, Corwell, Chen, Hallett, \& Cohen, 1997; Nachev, Kennard, \& Husain, 2008; Verwey, Lammens, \& van Honk, 2002). Heightened recruitment of the medial premotor areas is consistent with the claim based on behavioral work that practice with greater $\mathrm{CI}$ encourages the use of the motor chunking mode (Abrahamse et al., 2013; Immink \& Wright, 2001; Wright et al., 2004).

Random practice was also associated with a bilateral increase in activation in angular gyrus, superior parietal lobe, inferior temporal lobe, and precuneus across practice (Wymbs \& Grafton, 2009). These regions are important for the development and use of visual-spatial mappings for complex scenes that are central to the initial acquisition of motor sequences (Hikosaka et al., 1999, 2002). The formation of this type of representation for a motor sequence is associated with effectorindependent performance (Hikosaka et al., 2002). A few studies have revealed evidence for superior effector transfer performance following random practice (e.g., Smith \& Davies, 1995).
The findings that (1) much of the sequence-specific activation that differentiated practice condition reported by Wymbs and Grafton (2009) was bi-lateral, and (2) many of the active regions were pertinent to developing a representation of the motor sequence in visual-spatial coordinates, suggests that the performer was still at a relatively early point in learning (Dayan \& Cohen, 2011; Hikosaka et al., 1999). This being true, it is not surprising that Wymbs and Grafton also reported significant activation in the posterior lateral portion of the cerebellum (i.e., Crus II) during the high CI regime. This region of the cerebellum is the primary component of the "supramodal zone" and has reported connectivity with both dorsolateral prefrontal cortex and inferior parietal lobe (O'Reilly, Beckmann, Tomassini, Ramnani, \& Johansen-Berg, 2010). Moreover, it has been proposed that cortico-cerebellar circuits are important early in motor sequence learning but play a smaller role at later stages during which cortico-striatal circuits are more predominant (Doyon \& Benali, 2005). Both the cortico-cerebellar and cortico-striatal pathways have been implicated in attention orienting, which is consistent with $\mathrm{Li}$ and Wright's (2000) earlier report of greater attention demand during a high CI training format. In the case of blocked practice, Wymbs and Grafton reported that recruitment patterns during training involved a more restricted subset of neural regions for significantly less time, implying that less preparatory activity important for successful storage and retrieval of motor sequences occurred during low CI practice context.

Engagement of a broad range of key planning and executive control regions during random practice was confirmed by Lin et al. (2011). While this general conclusion appears robust, the specific neural regions recruited during random compared to blocked practice was far more limited compared to that reported by Wymbs and Grafton (2009). This may have been a function of Lin et al. (2011) describing average activation profiles across only 2 days of practice, noting where heightened activity was observed for random compared to blocked practice as opposed to dissecting neural activity more frequently across training (see Wymbs \& Grafton, 2009). Nonetheless, regions associated with executive control and building visual-spatial representations for each motor sequence again differentiated the practice conditions. For example, activity in regions crucial to recognition of objects in complex scenes (e.g., parahippocampal gyri, medial and inferior temporal lobe) as well as color coding and recognition were more prevalent during random practice, which would be expected given that each motor sequence used in Lin et al. was assigned a unique combination of colored stimuli. Finally, the dorsal premotor area and SMA as well as the postcentral gyrus (i.e., S1), all previously described as critical for the orderly planning of sequential actions, were again recruited more extensively during the high CI practice case (Cross et al., 2007; Wymbs \& Grafton, 2009). 
A significant contribution from contra-lateral dorsolateral prefrontal cortex for younger adults during random practice, not highlighted in previous work (Lin et al., 2011), was noted in a subsequent study (Lin et al., 2012). This was in contrast to older participants, who also benefitted from random practice, but adopted a different neural strategy during acquisition that involved recruiting right rostral prefrontal cortex, SMA, and M1. The right rostral prefrontal cortex has been linked to retrieval of episodic memories (Okuda et al., 2007). Lin et al. (2012) proposed that given the heightened cognitive demand in random training, older adults resort to retrieving task-relevant information about each motor sequence from long-term memory whereas younger adults maintain requisite information from trial to trial in working memory, requiring greater recruitment of dorsolateral prefrontal cortex. These data suggest that random practice can induce the use of alternative motor planning strategies, each of which are sufficient to support learning. Moreover, the data from Lin et al. (2012) raise questions as to the efficacy of a claim from the elaboration position that information about multiple tasks must coexist in working memory to observe benefits from random practice (Lin et al., 2012).

In summary, it appears clear that random practice instigates the recruitment of a broader network of neural regions that have previously been identified as critical for establishing motor memories (Dayan \& Cohen, 2011; Doyon et al., 2009; Floyer-Lea \& Matthews, 2005; Hardwick et al., 2013; Penhune \& Steele, 2012). One additional finding, however, is noteworthy as it highlights that the differential cognitive activity prompted by varying levels of $\mathrm{CI}$ is not just restricted to the recruitment of key motor planning sites. Individuals during blocked practice exhibited extensive activation in medial prefrontal cortex, left posterior cingulate gyrus, and the

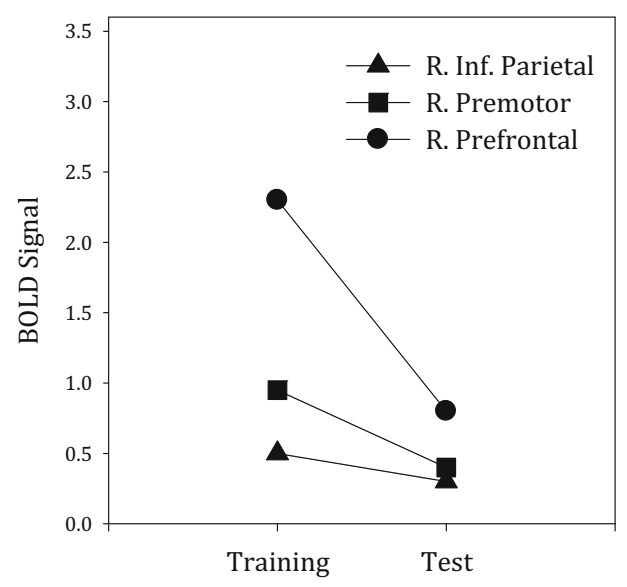

Blocked Practice

Fig. 4 Activation (bold signal) of right inferior parietal (triangle), right premotor (square), and right prefrontal (circles) regions for individuals assigned to blocked (black symbols) and random (white symbols) training formats. Importantly, the recruitment of each of these sites is greater for participants assigned to random practice during the training phase. This retrospineal anterior cingulate cortex. Parts of the prefrontal cortex, cingulate cortex, and the posterior parietal lobe have been considered components of the default or scaffolding network thought to facilitate the handling of either novel or complex tasks (Kelly \& Garavan, 2005; Petersen, van Mier, Fiez, \& Raichle, 1998). The default network is most active when individuals participate in undisturbed thinking or when not engaged in evaluation of the external world (Buckner, Andrews-Hanna, \& Schacter, 2008). Interestingly, in contrast to the subjects faced with blocked practice, individuals in random practice exhibited rapid reduction in the activation of the default network early in practice. This finding intimates a degree of efficiency in the cognitive activity employed by individuals during random practice that is not present for their blocked practice counterparts. That is, the high CI practice environment quickly forces the learner to minimize nonsequence related processing and instead allocate attention to recruiting motor planning regions that are associated with the development of resilient motor memories.

To this point, the focus has been on unique neural recruitment occurring during the acquisition phase of a blocked and random practice condition. A novel contribution from Lin et al. (2011) was to delineate the neural sites recruited during blocked and random delayed tests as a result of being exposed to different practice schedules during training. This was absent in Wymbs and Grafton (2009). During the test, not only did blocked practice participants display the anticipated slower response times for sequence production, but this was accomplished by activating the same motor sequence planning network that their random practice counterparts adopted during the training phase and continued to use, at a reduced level, during the test (see Fig. 4). In this work, these regions included contralateral prefrontal cortex and premotor areas as well as the

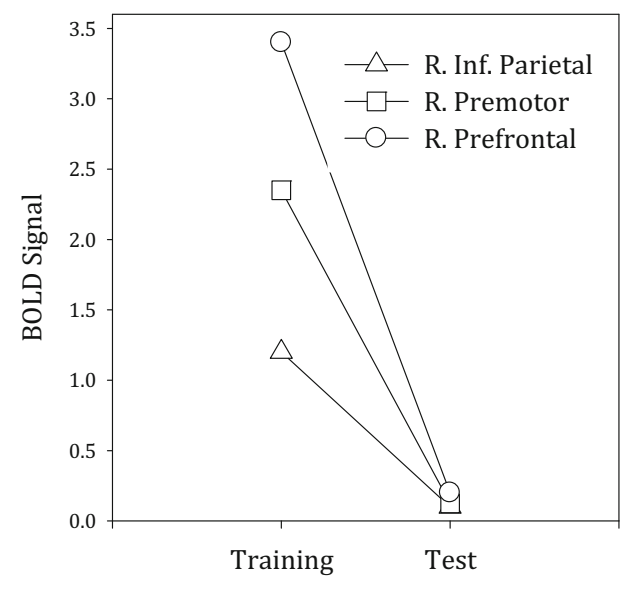

\section{Random Practice}

profile is reversed at the time of test with participants that experienced blocked practice now revealing greater recruitment of these key motor planning sites during the delayed test trials. Data adapted from Lin et al. (2011) 
inferior parietal lobe. The decreased recruitment of prefrontal, premotor, inferior parietal regions by the random practice participants during the test trials is especially important because it supports the claim that consolidation of motor sequence knowledge during, and possibly following, random practice occurs, leading to migration of sequence knowledge to more permanent storage sites for motor memories, such as M1 (Penhune \& Steele, 2012). Storing motor chunks developed during random practice might be one outcome that reduces the need to recruit such an elaborate set of motor planning sites to support motor sequence production during delayed tests (Immink \& Wright, 2001; Lin et al., 2011; Wright et al., 2004).

The neurophysiologic findings that have recently accompanied studies addressing CI during motor learning provide support for the core claim of the elaboration and reconstruction accounts that incorporating greater CI during practice results in a broader recruitment of neural sites that were identified previously as important contributors to successful longterm implementation of motor sequences (Cross et al., 2007; Dayan \& Cohen, 2011; Doyon et al., 2009; Lin et al., 2012; Lin et al., 2011; Penhune \& Steele, 2012; Wymbs \& Grafton, 2009). Despite this, the findings of Lin et al. $(2011,2012)$ in conjunction with those of Grafton and colleagues (Cross et al., 2007; Wymbs \& Grafton, 2009) do little in the way of uncovering a specific neural profile that might act as a signature to differentiate low and high CI training, if such a profile exists. Nonetheless, in the few studies that examined the neural underpinnings of the CI effect directly (Cross et al., 2007; Lin et al., 2011; Wymbs \& Grafton, 2009), a few sites consistently emerged revealing more activity during random rather than blocked practice (see Table 1). Specifically, there is a frequent, and reliable, presence of heightened activation in the medial PM region, including pSMA - a neural region reported to be important in the acquisition of discrete motor sequences (Kennerley, Sakai, \& Rushworth, 2004). The importance of this site is further verified by the finding that retention performance has been shown to be highly correlated with greater recruitment of contralateral SMA and M1 during

Table 1 Neural regions that have been identified as being more extensively recruited during the preparation of movement sequences during random as opposed to blocked practice in studies that have addressed the CI effect using fMRI. At least three of these (pSMA, random practice (Lin et al., 2012; Lin et al., 2011). The next section takes a closer look at the SMA complex and its importance to fostering the development of resilient motor chunks in a high CI practice context.

\section{Motor chunk development through recruitment of the SMA complex is an important outcome from experiencing greater contextual interference in practice}

Early and heightened SMA complex activity as well as the development of resilient motor chunks is associated with random practice (see Table 1) (Immink \& Wright, 2001; Lin et al., 2011; Wright et al., 2004; Wymbs \& Grafton, 2009). This relationship is interesting given that the SMA complex, consisting of SMA proper and pSMA, has been associated with diverse roles during the learning of sequential behaviors (Nachev et al., 2008). While not within the confines of experiments directly addressing CI but from studies addressing sequence learning per se, two specific responsibilities have been associated with the SMA complex, namely (1) supervising the switching between or inhibition of available action plans (Rushworth, Walton, Kennerley, \& Bannerman, 2004), and (2) supervising the formation of response-to-response action sets for complex sequential behaviors (Kennerley et al., 2004). Switching and inhibitory processes would presumably occur more frequently during high CI practice and might therefore account for the additional SMA complex recruitment during random as opposed to blocked practice (Lin et al., 2011; Wymbs \& Grafton, 2009). However, Rushworth et al. (2004) reported that the switching costs involving SMA result from shifts between arbitrary rules that dictate the nature of the stimulus-response (S-R) associations being used by the learner to perform an upcoming task. Given that S-R associations do not typically differ across practice schedules in most CI studies (i.e., the stimuli used to prompt the production of a particular action are fixed across practice), it is unlikely that heightened SMA complex activity in random training results from this demand.

\begin{tabular}{llll}
\hline Region & Functional name & Side & Reference \\
\hline Superior frontal gyrus & pSMA & $\mathrm{R}$ & Wymbs \& Grafton (2009), Lin et al. (2011) \\
Superior frontal gyrus & pSMA & L & Wymbs \& Grafton (2009) \\
Precentral gyrus & PMd & R & Wymbs \& Grafton (2009), Lin et al. (2011), Cross et al. (2007) \\
Precentral gyrus & PMd & L & Wymbs \& Grafton (2009) \\
Postcentral gyrus & S1 & R & Lin et al. (2011), Cross et al. (2007) \\
Postcentral gyrus & S1 & L & Wymbs \& Grafton (2009) \\
Inferior temporal gyrus & ITG & R & Wymbs \& Grafton (2009), Lin et al. (2011) \\
\hline
\end{tabular}

PMd, and S1) are considered part of the motor learning network used during the early acquisition of a motor sequence. $p S M A$ preSupplementary motor area, $P M d$ dorsal component of premotor area, $S 1$ somatosensory cortex, $I T G$ inferior temporal gyrus 
A second function of the SMA complex that is linked to governing the implementation of complex sequential actions across practice appears more relevant here. For example, Nakamura, Sakai, and Hikosaka (1999), using a monkey model, reported that inactivating pSMA but not SMA proper (using injections of muscimol) had a detrimental effect on learning new but not previously acquired motor sequences. This work specifically identified pSMA as central to the development and use of motor chunks that involved linking individual motor elements in the correct serial order. This claim was substantiated by Kennerley, Sakai, and Rushworth (2004) for human motor behavior by pairing TMS with the learning of a complex motor sequence. Following practice of this sequence, applying TMS to pSMA induced poorer sequence performance when applied at the time at which a motor chunk was initiated. This was not the case if TMS was applied while executing an element that was an internal part of a motor chunk. The works of Nakamura et al. and Kennerley et al. are in agreement with the claim of Rushworth et al. (2004) that pSMA is central to establishing an action set that comprises an order of responseresponse associations directing the selection of elements contained within a sequential behavior. The development of response-response associations is a hallmark of the "motor chunk" that is central to recent theorizing regarding the process of learning a single motor sequence (see Fig. $4 \mathrm{c}$ adapted from Abrahamse et al., 2013). One possibility then is that the greater SMA complex recruitment in the high CI practice context occurs in response to the establishment of motor chunks being readied to be stored permanently either during or shortly after practice. This claim is congruent with evidence from behavioral work indicating that the chunking mode is adopted more permanently in random practice (Abrahamse et al., 2013; Rushworth et al., 2004).

The involvement of the SMA complex during different practice conditions is based almost entirely on recent neuroimaging data that has revealed a significant relationship between retention performance following random practice and recruitment of this neural region during acquisition (Lin et al., 2012; Lin et al., 2011; Wymbs \& Grafton, 2009). The only case in which the SMA complex was manipulated directly involved the use of low frequency $(1 \mathrm{~Hz})$ repetitive TMS (rTMS) during different CI training conditions to create a "virtual lesion" at this neural region (Tanaka, Honda, Hanakawa, \& Cohen, 2010). Specifically, participants were administered $10-15$ min of rTMS over SMA proper or received sham stimulation (i.e., control condition in which the virtual lesion is not created) shortly after random and blocked practice of three unique motor sequences. A test performed on day 2 revealed that the sham stimulation condition did little to change the typical CI effect with random practice participants displaying significantly faster sequence response times compared to their blocked practice counterparts. However, following rTMS at SMA proper, individuals trained in a blocked schedule exhibited roughly a $100 \%$ increase in sequence response times compared to its sham counterpart, which in turn had slower sequence response times than the random practice group. When the application of rTMS was delayed until $6 \mathrm{~h}$ after blocked practice, test performance was similar to that observed for the sham condition but still poorer than that observed for individuals given random practice. Tanaka et al. proposed that during the time interval between $10 \mathrm{~min}$ to 6 -h following blocked practice SMA proper plays an important role in stabilization of motor memories when training is scheduled in this manner. Conversely, disruption of SMA proper shortly after random practice failed to inhibit motor memory stabilization possibly because this practice format supports (1) a more rapidly storage of knowledge in SMA (i.e., during ongoing practice), or (2) more rapid migration of this knowledge to other important neural sites, such as striatum, parietal cortex, and/or M1 that are important neural regions for advanced learning (Doyon et al., 2009; Doyon \& Benali, 2005).

Neurophysiological tools have been used to identify SMA complex as a neural region integral to the development and implementation of motor chunks during motor sequence acquisition (Kennerley et al., 2004). Initial evidence reveals prolonged activation of SMA complex when acquiring multiple motor sequences in a random but not blocked practice format (Lin et al., 2011; Wymbs \& Grafton, 2009). Behavioral and neurophysiologic evidence then converge on the possibility that more extensive recruitment of the SMA complex is associated with motor chunk development and may be a distinguishing characteristic of low and high CI training contexts. Unfortunately, at this juncture, the extant data does little in the way of isolating specific roles for SMA proper and pSMA during high CI training. A more precise description of the unique contributions of each constituent of this region, during random and blocked practice, would seem critical but awaits more thorough assessment. While the prevailing data implicates motor chunk development as a distinct benefit from high CI training, it would be premature to dismiss the possibility that random practice may also facilitate switching and inhibition processes that are likely more abundant during high $\mathrm{CI}$ training and as such may account for some SMA participation in this practice format. Clearly this issue is an important one that requires careful examination going forward. This is especially true given unpublished data from Wright et al. (2003) that suggests that greater CI in practice can mediate response selection skill, the complexity of which was altered by manipulating ongoing S-R association rules across training that were used to guide selection (see also, Pashler \& Baylis, 1991; Rushworth et al., 2004). 


\section{Relatively permanent adaptations are induced in motor circuits important for long-term motor performance through exposure to a high contextual interference practice context}

In the previous section it was proposed that, as a result of a more rigorous recruitment of the SMA complex during random compared to blocked practice that (1) more resilient motor chunks are developed (Immink \& Wright, 2001; Lin et al., 2012; Lin et al., 2011; Wright et al., 2004; Wymbs \& Grafton, 2009), and (2) that the motor chunks undergo rapid consolidation for storage in more permanent storage locations for motor memories (Lin et al., 2011; Tanaka et al., 2010). In the following sections additional evidence consistent with point (2) is offered as well as data suggesting that (3) critical adaptation in the neural architecture is also instigated by exposure to greater $\mathrm{CI}$ in practice. The adaptations described for point (3) are crucial because they afford rapid access to the new acquired motor sequence knowledge with less reliance on the use of critical motor planning sites (see, Lin et al., 2012; Lin et al., 2011).

\section{Consolidating motor sequence knowledge by rapid migration to permanent sites of storage through random practice}

There is considerable evidence implicating M1 as a neural site or as part of key neural networks critical to the permanent storage for motor memories (Penhune \& Steele, 2012). This claim is made at least in part on the basis that consolidation of motor sequence knowledge is disrupted or facilitated via application of rTMS or transcranial direct current stimulation over M1 immediately following extended practice (Boyd \& Linsdell, 2009; Muellbacher et al., 2002). Moreover, and important for the present discussion, there is evidence indicating the existence of practice-dependent plasticity in M1. For example, reliable adaptation has been revealed at M1 following 15 months of music training, the changes of which were correlated with behavioral improvements indexed through performance of motor and auditory versions of musical tests (Hyde et al., 2009). More recently, it has been demonstrated that even when extended practice with a motor sequence failed to induce detectable changes in the mean magnitude of neural activation in M1, additional practice still supported the utilization of stable patterns of activation at M1 that were not present for an unlearned motor sequence or for execution of the trained sequence with the untrained hand (Huang et al., 2013).

With respect to the CI effect, recall that Tanaka et al. (2010) argued that the resistance of sequence performance during random practice to the application of rTMS at SMA proper may result from crucial motor sequence knowledge being migrated more rapidly to neural sites or networks that include M1. Sizeable offline learning has been revealed following random as opposed to blocked practice, the magnitude of which was correlated positively with activation in ipsilateral M1 (Cross et al., 2007; Wymbs \& Grafton, 2009). More recently, Cohen, Cross, Wymbs, and Grafton (2009) demonstrated directly that ipsilateral M1 recruitment is crucial to consolidation of motor sequence knowledge. In this experiment, participants were administered two TMS pulses, separated by $100 \mathrm{~ms}$, over ipsilateral M1 during motor sequence preparation. Test performance of the sequences acquired while TMS was administered revealed significantly less overnight consolidation of motor sequence planning capability compared to when sequences were learned in the absence of the TMS manipulation. Thus, ipsilateral M1 is important for consolidation of motor sequence preparation and is independent of the well-documented role of M1 for sequence execution. Importantly, for the purposes of the present thesis, this study involved practice of the motor sequences in a random practice format. These data then are in keeping with the general claim that random practice can support rapid migration of important motor sequence knowledge to M1. However, some caution with this interpretation is necessary in light of Cohen et al.'s failure to include a blocked practice condition in this study (Cohen, Cross, Wymbs, \& Grafton, 2009). This is an important shortcoming that needs to be resolved before accepting the influence of high CI for consolidation of motor sequence knowledge to neural networks incorporating ipsilateral M1.

\section{Random practice improves accessibility to permanent motor sequence knowledge by inducing a long-term increase excitability in M1}

Increasing $\mathrm{CI}$ in practice is associated with changes in the neural architecture that affords efficient access to newly acquired sequence knowledge. Consistent with that claim are data revealing relatively long-term changes in excitability of M1 as a result of being privy to random but not blocked practice. We have discussed at length the work of Lin et al. $(2011,2012)$ in earlier sections when detailing neural activation patterns that emerge during different $\mathrm{CI}$ training conditions. In addition to the behavioral and neuroimaging data, Lin et al. (2011) also used paired-pulse TMS (ppTMS) during 2 days of random and blocked practice to assess concomitant changes in M1 excitability. Specifically, M1 excitability was indexed at $\mathrm{M} 1$ at the beginning and end of each of 2 days of training as well as prior to the test conducted on a fifth day. Paired pulse TMS involves the rapid presentation of two pulses, a conditioning and test stimulus, separated by varying inter-stimulus intervals (ISI) and is used to index the intracortical excitability. According to Lin et al. (2011) using short ISIs, in the order of $2-5 \mathrm{~ms}$, affords evaluation of intra-cortical inhibition. In contrast, the application of longer ISIs, in the range of 10-15 ms, allows assessment of the efficacy of intracortical facilitation (see also, Chen et al., 1998). 
Both short-term (within a single-practice session) and more permanent $(\sim 72 \mathrm{~h}$ ) adaptations in intra-cortical activity at M1 for individuals exposed to random but not blocked practice have been reported (Lin et al., 2012; Lin et al., 2011). The short-term benefit emerged after 2 days of random practice suggesting that some exposure to $\mathrm{CI}$ is needed to instigate even temporary shifts in M1 excitability. On the 5th day, when the test was conducted, only random practice participants exhibited reliably greater M1 activation from that observed at the beginning of the practice on day 2 . This finding is critical because MI intra-cortical excitability was only determined prior to practice on day 5, which means the heightened M1 activity persisted for at least a period of $72 \mathrm{~h}$ following random practice. Also important was Lin et al.'s observation that heightened activity in M1 during practice was highly correlated with superior performance during delayed retention tests. This form of practice-dependent plasticity in M1 appears quite robust having been demonstrated for both young (Lin et al., 2011) and older adults (Lin et al., 2012) exposed to random practice. It has been argued that greater M1 excitability during and following random practice is indicative of greater memory retrieval capacity (Lin et al., 2012; Lin et al., 2011).

\section{Random practice improves accessibility to permanent motor sequence knowledge by influencing functional and structural connectivity}

While considerable experimental endeavor geared toward understanding motor sequence learning focuses on the role of $\mathrm{M} 1$, it is becoming increasingly apparent that the development of functional neural networks or temporary neural connectivity is a hallmark of skilled sequential behavior. For example, there is general consensus that an advanced stage of motor sequence learning involves a transition from involvement of associative or antero-medial striatum to sensorimotor or dorsolateral striatum (Dayan \& Cohen, 2011; Wymbs, Bassett, Mucha, Porter, \& Grafton, 2012). Indeed, Penhune and Steele (2012) have argued that the sensorimotor striatum is a part of a critical neural network for encoding motor associations within elements of motor sequences as well as critical for the development and use of motor chunks (Abrahamse et al., 2013).

Recently the first efforts to examine the impact of CI during practice for the establishment of inter-regional functional connectivity have emerged. For example, psycho-physiological interaction analysis of fMRI data reported in earlier work of Lin et al. $(2011,2012)$ examined the connectivity with two particular neural regions, contralateral dorsolateral prefrontal and premotor areas, that are frequently considered central to motor sequence acquisition (Lin et al., 2013). As predicted, high CI practice enhanced inter-regional coupling between these areas, which was accompanied by a concomitant reduction in BOLD signal (reported earlier in the section discussing recruitment patterns during retention tests) at sites that exhibited increased connectivity. Both the decreased BOLD signal and increased functional connectivity suggest greater efficiency and/or economy when planning a motor sequence during random practice. While this adaptation took a couple of practice days to emerge, greater CI during practice led to functional connectivity between dorsolateral prefrontal cortex and superior medial frontal regions, the SMA, caudate nucleus, as well as the inferior and superior parietal areas. This was also true for the premotor area which again showed practicedependent neural networks emerging with M1, the cerebellum, and parietal areas following some initial exposure to random practice.

Clearly these data compliment many of the findings using neural imaging that revealed exposure to greater $\mathrm{CI}$ in practice evokes the use of a broader cerebral network temporally connected to facilitate performance of novel motor sequences (Cross et al., 2007; Lin et al., 2011; Wymbs \& Grafton, 2009). Importantly, only dorsolateral prefrontal cortex, not premotor, connectivity was sustained through the 72-h delayed test. The surviving network was limited to regions central to strategic control (i.e., superior medial frontal area) and using motor chunks (i.e., SMA and caudate nucleus). These data indicate that practice involving greater CI results in a resilient adaptation in the connectivity between the strategic network and the sensorimotor network to facilitate successful retrieval of the components of well-practiced motor sequences (Lin et al., 2013). Moreover, these findings are consistent with the claim that random practice does more than merely demand a broader range of planning processes, but more critically leads to permanent changes in the memory architecture that supports long-term motor sequence performance.

An alternative technology, diffusion-weighted magnetic resonance imaging (DWI), was used to evaluate white matter integrity following practice with varying levels of CI (Song et al., 2012). DWI is a brain imaging technique that uses fractional anisotropy (FA) to evaluate white matter integrity and structural connectivity strength of specific white matter tracts (Johansen-Berg, 2010; Johansen-Berg \& Rushworth, 2009). Participants in the study of Song et al. acquired a relatively long motor sequence (i.e., 12 elements) in a random or grouped (i.e., blocked) practice scheme. As expected, individuals exposed to random practice demonstrated reliably better performance immediately after training was completed, which was further sustained across a 1-week period.

The unique contribution of the work of Song et al. was the FA data, which was separately correlated with skilled performance at 1 week for each practice condition to determine the relationship between key white matter micro structural connectivity and performance. For blocked practice participants, performance of the motor sequence was correlated with FA in a section of a cortico-striatal tract that links lateral prefrontal cortex with anterior putamen. The anterior putamen is part of 
the associative striatum which previous neuroimaging studies have revealed to be important during the early learning of motor sequences (Dayan \& Cohen, 2011; Wymbs et al., 2012). In addition, FA for a tract bridging prefrontal cortex regions bilaterally was also correlated highly with performance for blocked practice participants. White matter pathways that emerged as important for delayed motor sequence performance following blocked practice were constituents of neural networks typically engaged very early in the acquisition of a motor sequence.

In contrast, only FA in white matter connecting the contralateral sensorimotor cortex and the posterior putamen, part of the sensorimotor striatum, was associated with performance observed after random practice. As noted earlier, this pathway has been identified as crucial to long-term performance improvements from neuroimaging studies. Moreover, it has been suggested that this pathway is central to long-term storage of motor sequence memory (Dayan \& Cohen, 2011; Penhune \& Steele, 2012). It is important to note that Song et al. (2012) designed their study using DWI to evaluate the influence of CI during implicit learning of a set of motor sequences. As a result, one must view with caution the tempting assumption that the same set of outcomes would occur if multiple sequences were learned using explicit strategies. Nonetheless, the notion that both functional and structural adaptations might be fostered through a high CI practice context is in keeping with the general tenor of this section that this practice format provides neural adaptations designed to offer more efficient access to stored information.

Despite the range of experimental approaches used during CI studies addressed in this section, a compelling case emerges for the claim that experience with greater $\mathrm{CI}$ in practice leads to non-transitory modifications of neural circuitry that is important for long-term motor sequence preparation and execution. This included evidence revealing rapid migration of knowledge to long-term storage sites or networks that become functionally connected during high CI practice. This, coupled with heightened intra-cortical excitability of at least one of these sites (i.e., M1) as well as additional functional connectivity with dorsolateral prefrontal cortex for strategic use of the acquired knowledge, suggests that high CI practice is effective in establishing an intricate memory and retrieval network for motor sequence knowledge (Shea et al., 1985; Shea \& Zimny, 1983).

\section{Contextual interference and motor sequence learning: what we know and what we need to know going forward}

Our understanding of how exposure to more CI during practice of multiple motor skills benefits their eventual retention has relied heavily on two theoretical positions: the reconstruction hypothesis and the elaboration hypothesis, which were forwarded exclusively on the basis of early behavioral research (Lee \& Magill, 1985; Shea \& Zimny, 1983). More recently, behavioral studies of CI and motor learning have been coupled with contemporary neurophysiologic tools. Accepting the fact that much recent work relies on one particular form of learning, motor sequence learning, some important insights as to how greater CI during practice enhances an individual's long-term performance capacity have surfaced. The remaining sections of this review are devoted to (1) highlighting the most critical novel insights from recent work that advances our current understanding of how CI impacts motor sequence learning, and (2) describing novel experimental work that is needed to further clarify the influence of CI during the acquisition of motor skills.

\section{No specific behavioral or neural signature or profile during blocked and random training has emerged}

A closer examination of recent work provides ample evidence that, when faced with greater $\mathrm{CI}$, the preparation of a motor sequence is more extensive, involving a broad set of neural regions that have been identified as critical to the acquisition of sequential motor skills (Doyon, Penhune, \& Ungerleider, 2003; Hardwick et al., 2013; Hikosaka et al., 2002; Li \& Wright, 2000; Penhune \& Steele, 2012; Wymbs \& Grafton, 2009). Part of these data highlight some efficiency in neural recruitment during a high CI practice environment evidenced as activation of primarily task-relevant neural regions at the expense of activation of the default or scaffolding network thought to facilitate the handling of either novel or complex tasks (Cross et al., 2007; Kelly \& Garavan, 2005; Petersen et al., 1998; Wymbs \& Grafton, 2009). Further evidence of processing efficiency is accomplished at the time of test by individuals trained with random practice. This is exhibited as reduced involvement of key motor regions recruited in training because sequence production can be governed by information stored in other neural circuits involving M1 (see Fig. 3, p. 1765, Lin et al., 2011).

Beyond this, however, distinguishing specific planning processes or a precise timeline of involvement of such processes during blocked and random practice is difficult to delineate. This is despite the implication, from early theorizing, that very specific and/or distinct planning operations characterize a high CI practice schedule (Lee \& Magill, 1983, 1985; Shea \& Zimny, 1983; Shea \& Zimny, 1988). Thus, the emergence of a unique neural profile or signature during particular CI practice regimes has not been forthcoming. Indeed, counter to the implications emanating from early theoretical positions regarding the nature of the planning operations that occur during blocked and random practice, it has been reported that individuals faced with these practice formats can, for example, adopt similar motor programming strategies during extensive 
training yet perform very differently at delayed tests (Wright et al., 2004). Young and older adults can both benefit from exposure to greater CI, yet do this using fundamentally different approaches. Younger adults rely on extensive use of working memory, whereas older adults are more reliant on repeated access of long-term memory (Lin et al., 2012). It seems safe to conclude that the poor initial performance associated with a high CI practice schedule can result from the adoption of a variety of neural and/or behavioral processing strategies.

\section{There are neural regions obligated relatively earlier in random but not blocked practice that have functional significance}

Rather than attempt to identify and delineate the timeline of an orderly set of processes that differentiate random and blocked practice formats, a more parsimonious approach is to identify the neural regions obligated by practice with greater CI and consider the functional importance of these select regions for long-term retention. In other words, irrespective of the processing strategy followed by an individual during random practice, this approach pinpoints neural sites that are reliably recruited during random rather than blocked practice that might act as "triggers" for establishing less labile motor sequence knowledge. Given that M1 has been granted the status as the likely storage site for new motor memories, this site would seem an appropriate candidate (Penhune \& Steele, 2012). The potential importance of M1 during training that involved unique levels of CI was certainly recognized by Lin and colleagues (Lin et al., 2008) who applied singlepulse TMS over M1 for individuals experiencing random and blocked practice. We propose, however, that the importance of M1, for practice with high CI, may be better reflected not during training but during the test phase. Recall that most descriptions of motor sequence learning consider neural circuits involving M1 as more crucial at the "slow" stage of learning rather than during the early or initial period of acquisition (Dayan \& Cohen, 2011; Penhune \& Steele, 2012). If random practice does induce greater consolidation to neural circuits involving M1 (Lin et al., 2011; Song et al., 2012; Tanaka et al., 2010), using TMS to disrupt processing at M1 either after the completion of practice or at the time of test should be much more disruptive for random practice participants than if TMS was applied at M1 early in training.

With respect to the initial period of encoding of motor sequence knowledge, more anterior sites, typically reflecting the demand for attention and executive function, are thought to be important (Dayan \& Cohen, 2011; Penhune \& Steele, 2012). The present review revealed two neural regions that have been identified as early participants in the motor skill learning process that are reliably recruited at an earlier point during random practice rather than blocked practice: PMd and the SMA complex (especially pSMA) (see Table 1). In reviewing the possible implications of differential recruitment of PMd and SMA complex by individuals faced with random practice, it is important to note the limited data from studies that have combined neuroimaging tools, primarily fMRI, with the traditional CI paradigm. Clearly, the need for verification of the differential use of these regions during a bout of training is needed. Nonetheless, of the studies available, there is remarkable consistency of earlier and more consistent recruitment of PMd and the SMA complex in addition to S1 during random practice as opposed to blocked practice (Cross et al., 2007; Lin et al., 2011; Wymbs \& Grafton, 2009).

In the case of PMd, all studies included in Table 1 identified greater right PMd activation during random practice (Cross et al., 2007; Lin et al., 2011; Wymbs \& Grafton, 2009). A review of 70 motor learning studies involving neural imaging, reported functional lateralization of $\mathrm{PMd}$, with right $\mathrm{PMd}$ being particularly important for the acquisition of a serial reaction time task because this region plays a central role in the learning and storage of perceptual sequences (Hardwick et al., 2013). Representing a motor sequence on the basis of its perceptual demands has been identified as an initial step in motor sequence acquisition (Abrahamse et al., 2013; Hikosaka et al., 2002). The fact that the right PMd is recruited more prominently during random practice suggests this practice format affords rapid organization of motor sequences at the perceptual level (Blandin, Proteau, \& Alain, 1994; Wright, Li, \& Coady, 1997).

Some evidence also emerged implicating greater left PMd activity during random practice (Wymbs \& Grafton, 2009). This neural region is reported to be less specialized for motor learning specific to the serial reaction time task. Rather, the left PMd, in conjunction with S1, appears to be a key feature of a broader neural network designed to support more general features of motor learning. This claim is made based on the recruitment of PMd and S1 during the learning of a broad range of motor tasks distinct from the serial reaction time task and more akin to adaptation tasks that require learning of novel kinematics and dynamics (Dayan \& Cohen, 2011; Doyon et al., 2009; Hardwick et al., 2013). Thus experiencing greater CI in practice might offer some potential for inducing some general learning strategies beyond those needed to acquire the motor sequences experienced during a bout of practice. If this is the case, it is possible that inducing greater CI might offer some advantage even when learning different classes of motor skills such as motor adaptation that rely on the recruitment of distinct neural circuitry (Doyon et al., 2009). At this time, evidence for widespread efficacy of greater CI beyond motor sequence tasks is quite limited (Pauwels et al., 2014; Sekiya, 2006; Song et al., 2012; Tsutsui, Lee, \& Hodges, 1998). Examining the pervasiveness of the CI effect across skills that have diverse sensorimotor demands, such as visual/dynamic adaptation or bimanual coordination, warrants more rigorous experimental attention in the future. 
The SMA complex, in particular the pSMA, is a second neural region that has been reported to be more active during random compared to blocked practice (Lin et al., 2011; Wymbs \& Grafton, 2009). Evidence was presented implicating the SMA complex as instrumental in organizing the internal structure of motor sequences (Hardwick et al., 2013; Kennerley et al., 2004; Nachev et al., 2008; Rushworth et al., 2004). Moreover, pSMA has been identified as a key player in supervising the use of a spatial and/or motor representations for motor sequence production (Hikosaka et al., 1999). The elevated recruitment of the SMA complex during random practice likely facilitates the transition from representing a new motor sequence in a perceptual or allocentric scheme (i.e., associative mode, see Fig. 3) to a motor or egocentric scheme congruent with the development of motor chunks (Abrahamse et al., 2013; Wright et al., 2004). One possibility then is that the advanced engagement of PMd and SMA during random practice is tied intimately to imparting structure on the motor sequence (e.g., motor chunks), which is a pre-requisite for more permanent storage in M1.

The proposed role for PMd and SMA during random practice raises some interesting questions. For example, recall earlier that Lin and colleagues used TMS to disrupt the contribution of M1 involvement during early encoding (Lin et al., 2008). On the basis of the present account, adopting a similar strategy using TMS, to induce a disruption in the contribution of PMd and/or SMA rather than M1 during initial training, offers an opportunity to examine the differential role of these neural sites during different practice schedules. In this case, inhibiting the contribution of these regions independently or together would be expected to place a particular burden on the individuals experiencing random practice. If sufficient disruption was introduced, one would anticipate degradation in the expected performance returns from random practice.

Rather than disrupt an advantage present during random practice, an alternative strategy would be to focus on methods to circumvent the lack of sequence organization induced during blocked practice. For example, if extracting structure, via recruitment of PMd and SMA, is a functional benefit available through random practice, then highlighting potential structure for novel movement sequences should be particularly advantageous during blocked practice and possibly help minimize long-term benefits only observed following random practice during motor sequence learning. In most CI studies that involve motor sequence learning, the learner is not provided with any guidance as to how to organize the production of the task. However, there are numerous methods that can be introduced during training to encourage a very specific form of segmentation of a discrete sequence production task (Abrahamse et al., 2013). For example, introducing regularities in response order has been shown to foster the adoption of similar patterns of chunking across participants (Koch \&
Hoffmann, 2000; Povel \& Collard, 1982; Restle, 1970; Sakai et al., 2003). The insertion of a temporal pause between the presentations of two successive stimuli within a motor sequence prompts the learner to divide the sequence in to separate parts around the pause. This structure appears quite permanent as it emerges even for trials during which the pause is removed (De Kleine \& Verwey, 2009; Stadler, 1993; Verwey, 2010; Verwey \& Dronkert, 1996). Finally, different colored stimuli have been used to encourage learners to treat elements of a motor sequence as smaller units in a serial reaction time task (Jimenez, Mendez, Pasquali, Abrahamse, \& Verwey, 2011). That is, elements of the motor sequence designated by the same color stimuli were treated as part of the same unit. Any one of these methods offer the opportunity to examine the relationship between the emergence of sequence organization during random and blocked practice and resultant memory performance.

\section{Early recruitment of PMd and SMA complex during random practice facilitates access to stored motor sequence knowledge}

Evidence was offered linking the recruitment of SMA complex during random practice to the early development of motor chunks. Establishing motor chunks has been described as a fundamental strategy used to facilitate continued improvement in the performance of complex serial behaviors and considered central to motor skill learning (Sakai et al., 2003; Verwey \& Eikelboom, 2003). In addition to being involved with the establishment of resilient motor sequence knowledge during random practice, recruitment of the premotor region also appears to play a key role in ensuring effective access to the newly acquired information. For example, Lin et al. (2013) reported the emergence of functional coupling between right PMd and M1 after 2 days of random but not blocked practice. In addition to the emergence of this functional coupling is evidence of a concomitant increase in M1 excitability across random practice (Lin et al., 2012; Lin et al., 2011). Greater PMd-M1 connectivity as well as M1 excitability during practice are associated with superior learning displayed $72 \mathrm{~h}$ later.

The temporary linkage between PMd and M1 was not reliable at the time of test and suggests that this circuit's functional role is localized to encoding rather than retrieval. While the magnitude of coupling between PMd and M1 is reduced significantly at the time of test, M1 excitability continues to be elevated significantly following random practice. Presumably, this elevated excitability results from the earlier PMd connectivity, and the extent of excitability is highly predictive of the test benefit garnered from random practice (Lin et al., 2012; Lin et al., 2011). This is congruent with the claim that M1 has an important part to play in the eventual storage of motor 
sequence information and random practice facilitates this process via differential recruitment of PMd.

The role of PMd appears to be restricted to early encoding during random practice, as a result of being related to representing the novel motor sequence in perceptual space. The SMA complex has also been identified as an important component of a functional neural network with dorsal lateral prefrontal cortex that develops across practice. Unlike the aforementioned circuit involving PMd-M1, connectivity between dorsolateral prefrontal cortex and SMA remains important at the time of test with greater connectivity being correlated with the increased learning benefit from random practice (Lin et al., 2013). Presumably, developing response-response associations between elements would be part of operating at the chunking mode during practice (Abrahamse et al., 2013). As a consequence of using the chunking mode, motor chunks would develop, and continue to be important as execution of the motor sequence becomes internally driven (i.e., not being dependent on the presence of an external stimulus to predict the next response in sequence). It is entirely feasible that dorsolateral prefrontal cortex, given its reported role for attention allocation and task-switching, in conjunction with SMA, oversees this process during practice and at retention. If this claim is true, application of TMS to disrupt the contribution of dorsolateral prefrontal cortex at the time of testing, rather than during training, should be effective at reducing the random practice learning advantage. To date, we know of only one attempt to interfere with dorsolateral prefrontal cortex during motor sequence learning but this study addressed practice variability and involved application of TMS just after training was completed (Kantak, Sullivan, Fisher, Knowlton, \& Winstein, 2010, 2011).

\section{Other relatively permanent changes in neural architecture critical for the storage and retrieval of motor sequence knowledge appear possible following random practice but need additional experimental verification}

The functional connectivity described in the previous section is a temporary form of adaption in the neural architecture that reduces the reliance on the availability of an external stimulus to dictate sequence production as a result of being trained within the confines of greater CI. A byproduct of these changes are other efficiencies such as reduced need to activate key motor planning sites that were recruited during acquisition in random practice (e.g., right PMd) at retention. Indeed, Lin et al. (2011) reported that the size of the learning effect resulting from random practice was inversely proportional to the reduction in BOLD signal in neural sites related to executive function and movement planning (see Fig. 5 in Lin et al., 2011).

More recently, with the advent of DWI, adaptation resulting from random practice has extended beyond functional connectivity to structural connectivity. White matter microstructural correlates of long-term skill development following random and blocked practice were recently described for the first time (Song et al., 2012). A noteworthy observation was that random practice was associated with improved integrity of the cortico-striatal tract connecting sensorimotor cortex and posterior putamen. This is interesting for a couple of reasons. First, there is general consensus that motor skill learning involves a shift in reliance from associative to sensorimotor striatum (Coynel et al., 2010; Dayan \& Cohen, 2011; Lehericy et al., 2005; Penhune \& Steele, 2012), which is thought to contribute to the acquisition of a motor representation of a sequential behavior (Hikosaka et al., 1999) possibly involving the development of motor chunks (Penhune \& Steele, 2012; Wymbs et al., 2012). Random practice seems to support this crucial feature of the learning process more effectively than blocked practice and there is behavioral data consistent with this claim (Wright et al., 2004). Second, from a more historical perspective, the connectivity data appear remarkably congruent with the basic tenet of the elaboration position that highlighted the importance of the development of an effective retrieval network as a result of a high CI practice schedule. These data then provide some needed detail that was noted earlier as lacking in the initial account describing how a more "elaborate" memory network might be implemented within the neural architecture.

Combining behavioral work with neurophysiologic methods to assess the underlying neural adaptations associated with exposure to various levels of CI during practice is in its infancy so many of the initial findings reported herein need to be verified. Nonetheless, the initial experimental efforts, especially those pertaining to functional connectivity, discussed in this section are consistent with the claim that random practice can induce widespread adaption in the neural apparatus that is central to storage and efficient retrieval of motor sequence knowledge. With respect to the inducement of structural adaptation through high CI training, work focusing on white matter integrity is in its infancy but promising (Song et al., 2012). However, the need for additional experimental work using DWI is particularly important given Song et al. (2012) focused only on implicit motor sequence learning. It is also possible that random practice might not be restricted to stimulating adaptation in white matter but may also contribute to practice-dependent plasticity that has been demonstrated at M1 during the acquisition of single motor sequences (Karni et al., 1995; Pascual-Leone et al., 1995). Specifically, if, as suggested earlier in this section, M1 plays a central role in the storage of motor sequence knowledge, one might expect to see this reflected in additional structural changes at M1 following random but not blocked practice. With respect to M1 plasticity, Karni et al. (1995) have shown persistence of this form of adaptation, when learning single motor sequences, to be in the order of a few months. It would 
be interesting to evaluate if the practice schedule to which the learner is exposed mediates the time course of persistence of such plasticity.

Acknowledgment The present work was supported in part by a grant from the Science Foundation of the Ministry of Education of China (Grant No.13YJC890006) awarded to C.J.

\section{References}

Abrahamse, E. L., Ruitenberg, M. F. L., de Kleine, E., \& Verwey, W. B. (2013). Control of automated behavior: insights from the discrete sequence production task. Frontiers in Human Neuroscience, 7, 118.

Adams, S. G., \& Page, A. D. (2000). Effects of selected practice and feedback variables on speech motor learning. Journal of Medical Speech-Language Pathology, 8(4), 215-220.

Black, C. B., Wright, D. L., Magnuson, C. E., \& Brueckner, S. (2005). Learning to detect error in movement timing using physical and observational practice. Research Quarterly for Exercise and Sport, 76(1), 28-41.

Blandin, Y., Proteau, L., \& Alain, C. (1994). On the cognitive-processes underlying contextual interference and observational-learning. Journal of Motor Behavior, 26(1), 18-26.

Boyd, L. A., \& Linsdell, M. A. (2009). Excitatory repetitive transcranial magnetic stimulation to left dorsal premotor cortex enhances motor consolidation of new skills. BMC Neuroscience, 10, 72. doi:10. 1186/1471-2202-10-72

Brady, F. (1998). A theoretical and empirical review of the contextual interference effect and the learning of motor skills. Quest, 50(3), 266-293.

Brady, F. (2004). Contextual interference: A meta-analytic study. Perceptual and Motor Skills, 99(1), 116-126. doi:10.2466/pms.99. 1.116-126

Brady, F. (2008). The contextual interference effect and sport skills. Perceptual and Motor Skills, 106(2), 461-472. doi:10.2466/Pms. 106.2.461-472

Buchanan, J. J., \& Wright, D. L. (2011). Generalization of action knowledge following observational learning. Acta Psychologica, 136(1), $167-178$.

Buchanan, J. J., Zihlman, K., Ryu, Y. U., \& Wright, D. L. (2007). Learning and transfer of a relative phase pattern and a joint amplitude ratio in a rhythmic multijoint arm movement. Journal of Motor Behavior, 39(1), 49-67.

Buckner, R. L., Andrews-Hanna, J. R., \& Schacter, D. L. (2008). The brain's default network - anatomy, function, and relevance to disease. Annals of the New York Academy of Sciences, 1124(1), 1-38. doi:10.1196/annals. 1440.011

Carlson, R. A., \& Yaure, R. G. (1990). Practice schedules and the use of component skills in problem-solving. Journal of Experimental Psychology-Learning Memory and Cognition, 16(3), 484-496. doi:10.1037/0278-7393.16.3.484

Carlson, R. A., Khoo, B. H., Yaure, R. G., \& Schneider, W. (1990). Acquisition of a problem-solving skill-levels of organization and use of working memory. Journal of Experimental PsychologyGenera, 119(2), 193-214. doi:10.1037/0096-3445.119.2.193

Carroll, W. R., \& Bandura, A. (1982). The role of visual monitoring in observational-learning of action patterns - making the unobservable observable. Journal of Motor Behavior, 14(2), 153-167.

Carroll, W. R., \& Bandura, A. (1990). Representational guidance of action production in observational-learning - a causal-analysis. Journal of Motor Behavior, 22(1), 85-97.
Chen, R., Tam, A., Butefisch, C., Corwell, B., Ziemann, U., Rothwell, J. C., \& Cohen, L. G. (1998). Intracortical inhibition and facilitation in different representations of the human motor cortex. Journal of Neurophysiology, 80(6), 2870-2881.

Cohen, N. R., Cross, E. S., Wymbs, N. F., \& Grafton, S. T. (2009). Transient disruption of M1 during response planning impairs subsequent offline consolidation. Experimental Brain Research, 196(2), 303-309. doi:10.1007/s00221-009-1838-x

Coynel, D., Marrelec, G., Perlbarg, V., Pelegrini-Issac, M., Van de Moortele, P. F., Ugurbil, K., ... Lehericy, S. (2010). Dynamics of motor-related functional integration during motor sequence learning. Neuroimage, 49(1), 759-766. doi: 10.1016/j.neuroimage. 2009.08 .048

Cross, E. S., Schmitt, P. J., \& Grafton, S. T. (2007). Neural substrates of contextual interference during motor learning support a model of active preparation. Journal of Cognitive Neuroscience, 19(11), 1854-1871. doi:10.1162/jocn.2007.19.11.1854

Dayan, E., \& Cohen, L. G. (2011). Neuroplasticity subserving motor skill learning. Neuron, 72(3), 443-454. doi:10.1016/j.neuron.2011.10.008

de Croock, M. B. M., van Merrienboer, J. J. G., \& Paas, F. G. W. C. (1998). High versus low contextual interference in simulation-based training of troubleshooting skills: Effects on transfer performance and invested mental effort. Computers in Human Behavior, 14(2), 249-267. doi:10.1016/S0747-5632(98)00005-3

De Kleine, E., \& Verwey, W. B. (2009). Motor learning and chunking in dyslexia. Journal of Motor Behavior, 41(4), 331-337.

Del Rey, P. (1982). Effects of contextual interference on the memory of older females differing in levels of physical-activity. Perceptual and Motor Skills, 55(1), 171-180.

Destrebecqz, A., \& Cleeremans, A. (2001). Can sequence learning be implicit? New evidence with the process dissociation procedure. Psychonomic Bulletin \& Review, 8(2), 343-350.

Doyon, J., \& Benali, H. (2005). Reorganization and plasticity in the adult brain during learning of motor skills. Current Opinion in Neurobiology, 15(2), 161-167. doi:10.1016/j.conb.2005.03.004

Doyon, J., Penhune, V., \& Ungerleider, L. G. (2003). Distinct contribution of the cortico-striatal and cortico-cerebellar systems to motor skill learning. Neuropsychologia, 41(3), 252-262.

Doyon, J., Bellec, P., Amsel, R., Penhune, V., Monchi, O., Carrier, ... Benali, H. (2009). Contributions of the basal ganglia and functionally related brain structures to motor learning. Behavioral Brain Research, 199(1), 61-75. doi: 10.1016/j.bbr.2008.11.012

Dubrowski, A., Backstein, D., Abughaduma, R., Leidl, D., \& Carnahan, H. (2005). The influence of practice schedules in the learning of a complex bone-plating surgical task. American Journal of Surgery, 190(3), 359-363.

Fendrich, D. W., Healy, A. F., \& Bourne, L. E. (1991). Long-term repetition effects for motoric and perceptual procedures. Journal of Experimental Psychology-Learning Memory and Cognition, 17(1), $137-151$

Floyer-Lea, A., \& Matthews, P. M. (2005). Distinguishable brain activation networks for short- and long-term motor skill learning. Journal of Neurophysiology, 94(1), 512-518. doi:10.1152/jn.00717.2004

Gerloff, C., Corwell, B., Chen, R., Hallett, M., \& Cohen, L. G. (1997). Stimulation over the human supplementary motor area interferes with the organization of future elements in complex motor sequences. Brain, 120, 1587-1602. doi:10.1093/brain/120.9.1587

Goode, S., \& Magill, R. A. (1986). Contextual interference effects in learning 3 badminton serves. Research Quarterly for Exercise and Sport, 57(4), 308-314.

Grafton, S. T., Hazeltine, E., \& Ivry, R. (1995). Functional mapping of sequence learning in normal humans. Journal of Cognitive Neuroscience, 7(4), 497-510.

Hardwick, R. M., Rottschy, C., Miall, R. C., \& Eickhoff, S. B. (2013). A quantitative meta-analysis and review of motor learning in the human brain. NeuroImage, 67, 283-297. 
Hikosaka, O., Nakahara, H., Rand, M. K., Sakai, K., Lu, X. F., Nakamura, ... Doya, K. (1999). Parallel neural networks for learning sequential procedures. Trends in Neurosciences, 22(10), 464 471. doi: 10.1016/S0166-2236(99)01439-3

Hikosaka, O., Nakamura, K., Sakai, K., \& Nakahara, H. (2002). Central mechanisms of motor skill learning. Current Opinion in Neurobiology, 12(2), 217-222.

Huang, Y., Zonglei, Z., Song, Y., Zhu, Q., Wang, S., \& Liu, J. (2013). Motor training increases the stability of activation patterns in the primary motor cortex. PloS One, 8(1), e53555. doi:10.1371/ journal.pone.0053555

Hyde, K. L., Lerch, J., Norton, A., Forgeard, M., Winner, E., Evans, A. C., \& Schlaug, G. (2009). Musical training shapes structural brain development. Journal of Neuroscience, 29(10), 3019-3025. doi:10. 1523/Jneurosci.5118-08.2009

Immink, M. A., \& Wright, D. L. (1998). Contextual interference: a response planning account. Quarterly Journal of Experimental Psychology Section A-Human Experimental Psychology, 51(4), 735-754.

Immink, M. A., \& Wright, D. L. (2001). Motor programming during practice conditions high and low in contextual interference. Journal of Experimental Psychology-Human Perception and Performance, 27(2), 423-437. doi:10.1037//0096-1523.27.2.423

Jimenez, L., Mendez, A., Pasquali, A., Abrahamse, E., \& Verwey, W. (2011). Chunking by colors: assessing discrete learning in a continuous serial reaction-time task. Acta Psychologica, 137(3), 318-329.

Johansen-Berg, H. (2010). Behavioural relevance of variation in white matter microstructure. Current Opinion in Neurology, 23(4), 351358. doi:10.1097/Wco.0b013e32833b7631

Johansen-Berg, H., \& Rushworth, M. F. S. (2009). Using diffusion imaging to study human connectional anatomy. Annual Review of Neuroscience, 32, 75-94. doi:10.1146/annurev.neuro.051508. 135735

Kantak, S. S., Sullivan, K. J., Fisher, B. E., Knowlton, B. J., \& Winstein, C. J. (2010). Neural substrates of motor memory consolidation depend on practice structure. Nature Neuroscience, 13(8), 923-925.

Kantak, S. S., Sullivan, K. J., Fisher, B. E., Knowlton, B. J., \& Winstein, C. J. (2011). Transfer of motor learning engages specific neural substrates during motor memory consolidation dependent on the practice structure. Journal of Motor Behavior, 43(6), 499-507.

Karni, A., Meyer, G., Jezzard, P., Adams, M. M., Turner, R., \& Ungerleider, L. G. (1995). Functional MRI evidence for adult motor cortex plasticity during motor skill learning. Nature, 377(6545), 155-158.

Karni, A., Meyer, G., Rey-Hipolito, C., Jezzard, P., Adams, M. M., Turner, R., \& Ungerleider, L. G. (1998). The acquisition of skilled motor performance: fast and slow experience-driven changes in primary motor cortex. Proceedings of the National Academy of Sciences of the United States of America, 95(3), 861-868. doi:10. 1073/pnas.95.3.861

Kelly, A. M. C., \& Garavan, H. (2005). Human functional neuroimaging of brain changes associated with practice. Cerebral Cortex, 15(8), 1089-1102. doi:10.1093/cercor/bhi005

Kennerley, S. W., Sakai, K., \& Rushworth, M. F. S. (2004). Organization of action sequences and the role of the pre-SMA. Journal of Neurophysiology, 91(2), 978-993. doi:10.1152/jn.00651.2003

Klapp, S. T. (1995). Motor response programming during simple and choice-reaction time: The role of practice. Journal of Experimental Psychology-Human Perception and Performance, 21(5), 10151027. doi:10.1037//0096-1523.21.5.1015

Klapp, S. T. (1996). Reaction time analysis of central motor control. In H. N. Zelaznik (Ed.), Advance in Motor Learning and Control (pp. 1336). Champaign, IL: Human Kinetics.

Knock, T. R., Ballard, K. J., Robin, D. A., \& Schmidt, R. A. (2000). Influence of order of stimulus presentation on speech motor learning: A principled approach to treatment for apraxia of speech. Aphasiology, 14(5-6), 653-668.
Koch, I., \& Hoffmann, J. (2000). Patterns, chunks, and hierarchies in serial reaction-time tasks. Psychological Research-Psychologische Forschung, 63(1), 22-35. doi:10.1007/P100008165

Lee, T. D., \& Magill, R. A. (1983). The locus of contextual interference in motor-skill acquisition. Journal of Experimental PsychologyLearning Memory and Cognition, 9(4), 730-746. doi:10.1037// 0278-7393.9.4.730

Lee, T. D., \& Magill, R. A. (1985). Can forgetting facilitate skill acquisition? In D. Goodman, R. B. Wilberg, \& I. M. Franks (Eds.), Differing perspectives on memory, learning and control (pp. 322). Amsterdam: North Holland.

Lee, T. D., Wulf, G., \& Schmidt, R. A. (1992). Contextual interference in motor learning - dissociated effects due to the nature of task variations. Quarterly Journal of Experimental Psychology Section aHuman Experimental Psychology, 44(4), 627-644.

Lehericy, S., Benali, H., Van de Moortele, P. F., Pelegrini-Issac, M., Waechter, T., Ugurbil, K., \& Doyon, J. (2005). Distinct basal ganglia territories are engaged in early and advanced motor sequence learning. Proceedings of the National Academy of Sciences of the United States of America, 102(35), 12566-12571. doi:10.1073/pnas.0502762102

Li, Y. H., \& Wright, D. L. (2000). An assessment of the attention demands during random- and blocked-practice schedules. Quarterly Journal of Experimental Psychology Section a-Human Experimental Psychology, 53(2), 591-606. doi:10.1080/027249800390628

Lin, C. H., Fisher, B. E., Winstein, C. J., Wu, A. D., \& Gordon, J. (2008). Contextual interference effect: Elaborative processing or forgettingreconstruction? A post hoc analysis of transcranial magnetic stimulation-induced effects on motor learning. Journal of Motor Behavior, 40(6), 578-586. doi:10.3200/Jmbr.40.6.578-586

Lin, C. H., Winstein, C. J., Fisher, B. E., \& Wu, A. D. (2010). Neural correlates of the contextual interference effect in motor learning: a transcranial magnetic stimulation investigation. Journal of Motor Behavior, 42(4), 223-232.

Lin, C. H., Knowlton, B. J., Chiang, M. C., Iacoboni, M., Udompholkul, P., \& Wu, A. D. (2011). Brain-behavior correlates of optimizing learning through interleaved practice. NeuroImage, 56(3), 17581772. doi:10.1016/j.neuroimage.2011.02.066

Lin, C. H., Chiang, M. C., Wu, A. D., Iacoboni, M., Udompholkul, P., Yazdanshenas, O., \& Knowlton, B. J. (2012). Age related differences in the neural substrates of motor sequence learning after interleaved and repetitive practice. NeuroImage, 62(3), 2007-2020. doi: 10.1016/j.neuroimage.2012.05.015

Lin, C. H., Chiang, M. C., Knowlton, B. J., Iacoboni, M., Udompholkul, P., \& Wu, A. D. (2013). Interleaved practice enhances skill learning and the functional connectivity of fronto-parietal networks. Human Brain Mapping, 34(7), 1542-1558. doi:10.1002/Hbm.22009

Magill, R. A., \& Hall, K. G. (1990). A review of the contextual interference effect in motor skill acquisition. Human Movement Science, 9(3-5), 241-289. doi:10.1016/0167-9457(90)90005-X

Magnuson, C. E., \& Wright, D. L. (2004). Random practice can facilitate the learning of tasks that have different relative time structures. Research Quarterly for Exercise and Sport, 75(2), 197-202.

Magnuson, C. E., Wright, D. L., \& Verwey, W. B. (2004). Changes in the incidental context impacts search but not loading of the motor buffer. Quarterly Journal of Experimental Psychology Section AHuman Experimental Psychology, 57(5), 935-951.

Muellbacher, W., Ziemann, U., Wissel, J., Dang, N., Kofler, M., Facchini, S.,...Hallett, M. (2002). Early consolidation in human primary motor cortex. Nature, 415(6872), 640-644. doi: 10.1038/Nature712

Nachev, P., Kennard, C., \& Husain, M. (2008). Functional role of the supplementary and pre-supplementary motor areas. Nature Reviews Neuroscience, 9(11), 856-869. doi:10.1038/Nrn2478

Nakamura, K., Sakai, S., \& Hikosaka, O. (1999). Effects of local inactivation of monkey medial fronal cortex in learning of sequential procedures. Journal of Neurophysiology, 82, 1063-1068 
Nissen, M. J., \& Bullemer, P. (1987). Attentional requirements of learning - evidence from performance-measures. Cognitive Psychology, 19(1), 1-32.

Okuda, J., Fujii, T., Ohtake, H., Tsukiura, T., Yamadori, A., Frith, C. D., \& Burgess, P. W. (2007). Differential involvement of regions of rostral prefrontal cortex (Brodmann area 10) in time- and eventbased prospective memory. International Journal of Psychophysiology, 64(3), 233-246. doi:10.1016/j.ijpsycho.2006. 09.009

Ollis, S., Button, C., \& Fairweather, M. (2005). The influence of professional expertise and task complexity upon the potency of the contextual interference effect. Acta Psychologica, 118(3), 229-244. doi: 10.1016/j.actpsy.2004.08.003

O'Reilly, J. X., Beckmann, C. F., Tomassini, V., Ramnani, N., \& Johansen-Berg, H. (2010). Distinct and overlapping functional zones in the cerebellum defined by resting state functional connectivity. Cerebral Cortex, 20(4), 953-965. doi:10.1093/cercor/bhp157

Pascual-Leone, A., Dang, N., Cohen, L. G., Brasilneto, J. P., Cammarota, A., \& Hallett, M. (1995). Modulation of muscle responses evoked by transcranial magnetic stimulation during the acquisition of new fine motor-skills. Journal of Neurophysiology, 74(3), 1037-1045.

Pashler, H., \& Baylis, G. (1991). Procedural learning: 1. Locus of practice effects in speeded choice tasks. Journal of Experimental Psychology-Learning Memory and Cognition, 17(1), 20-32.

Pauwels, L., Swinnen, S. P., \& Beets, I. A. M. (2014). Contextual interference in complex bimanual skill learning leads to better skill persistence. Plos One, 9(6), e100906. doi:10.1371/journal.pone. 0100906

Penhune, V. B., \& Steele, C. J. (2012). Parallel contributions of cerebellar, striatal and M1 mechanisms to motor sequence learning. Behavioural Brain Research, 226(2), 579-591. doi:10.1016/j.bbr. 2011.09.044

Petersen, S. E., van Mier, H., Fiez, J. A., \& Raichle, M. E. (1998). The effects of practice on the functional anatomy of task performance. Proceedings of the National Academy of Sciences of the United States of America, 95(3), 853-860. doi:10.1073/pnas.95.3.853

Porretta, D. L., \& Obrien, K. (1991). The use of contextual interference trials by mildly mentally-handicapped children. Research Quarterly for Exercise and Sport, 62(2), 244-248.

Porter, J. M., \& Magill, R. A. (2010). Systematically increasing contextual interference is beneficial for learning sport skills. Journal of Sports Sciences, 28(12), 1277-1285. doi:10.1080/02640414.2010. 502946

Povel, D. J., \& Collard, R. (1982). Structural factors in patterned finger tapping. Acta Psychologica, 52(1-2), 107-123. doi:10.1016/00016918(82)90029-4

Rendell, M. A., Masters, R. S. W., \& Farrow, D. (2009). The paradoxical role of cognitive effort in contextual interference and implicit motor learning. International Journal of Sport Psychology, 40(4), 636647.

Restle, F. (1970). Theory of serial pattern learning - structural trees. Psychological Review, 77(6), 481-495.

Rosenbaum, D. A. (1980). Human movement initiation — specification of arm, direction, and extent. Journal of Experimental PsychologyGeneral, 109(4), 444-474.

Rushworth, M. F. S., Walton, M. E., Kennerley, S. W., \& Bannerman, D. M. (2004). Action sets and decisions in the medial frontal cortex. Trends in Cognitive Sciences, 8(9), 410-417. doi:10.1016/j.tics. 2004.07.009

Russell, D. M., \& Newell, K. M. (2007). How persistent and general is the contextual interference effect? Research Quarterly for Exercise and Sport, 78(4), 318-327.

Sakai, K., Kitaguchi, K., \& Hikosaka, O. (2003). Chunking during human visuomotor sequence learning. Experimental Brain Research, 152(2), 229-242.
Scully, D. M., \& Newell, K. M. (1985). Observational-learning and the acquisition of motor-skills: Toward a visual-perception perspective. Journal of Human Movement Studies, 11(4), 169-186.

Sekiya, H. (2006). Contextual interference in implicit and explicit motor learning. Perceptual and Motor Skills, 103(2), 333-343. doi:10. 2466/Pms.103.2.333-343

Shea, J. B., \& Morgan, R. L. (1979). Contextual interference effects on the acquisition, retention, and transfer of a motor skill. Journal of Experimental Psychology-Human Learning and Memory, 5(2), 179-187. doi:10.1037/0278-7393.5.2.179

Shea, J. B., \& Zimny, S. T. (1983). Context effects in memory and learning. In R. A. Magill (Ed.), Memory and Control of Action (pp. 145166). Amsterdam: North Holland.

Shea, J. B., \& Zimny, S. T. (1988). Knowledge incorporation in motor representation. In O. J. Meijer \& J. Roth (Eds.), Complex movement behaviour (pp. 345-366). Amsterdam: North Holland.

Shea, J. B., Hunt, J. P., \& Zimny, S. T. (1985). Representational structure and strategic processes for movement production. In D. Goodman, R. B. Wiberg, \& I. M. Frank (Eds.), Differing perspectives in motor learning, memory, and control (pp. 55-87). Amsterdam: Elsevier.

Smith, P. J. K., \& Davies, M. (1995). Applying contextual interference to the Pawlata roll. Journal of Sports Sciences, 13(6), 455-462. doi:10. 1080/02640419508732262

Song, S. B., Sharma, N., Buch, E. R., \& Cohen, L. G. (2012). White matter microstructural correlates of superior long-term skill gained implicitly under randomized practice. Cerebral Cortex, 22(7), 1671-1677. doi:10.1093/cercor/bhr247

Stadler, M. A. (1993). Implicit serial-learning - questions inspired by Hebb (1961). Memory \& Cognition, 21(6), 819-827.

Sternberg, S. (1969). Discovery of processing stages - extensions of Donders method. Acta Psychologica, 30, 276-315. doi:10.1016/ 0001-6918(69)90055-9

Tanaka, S., Honda, M., Hanakawa, T., \& Cohen, L. G. (2010). Differential contribution of the supplementary motor area to stabilization of a procedural motor skill acquired through different practice schedules. Cerebral Cortex, 20(9), 2114-2121. doi:10.1093/cercor/bhp276

Tsutsui, S., Lee, T. D., \& Hodges, N. J. (1998). Contextual interference in learning new patterns of bimanual coordination. Journal of Motor Behavior, 30(2), 151-157.

Verwey, W. B. (1999). Evidence for a multistage model of practice in a sequential movement task. Journal of Experimental PsychologyHuman Perception and Performance, 25(6), 1693-1708.

Verwey, W. B. (2010). Diminished motor skill development in elderly: Indications for limited motor chunk use. Acta Psychologica, 134(2), 206-214. doi:10.1016/j.actpsy.2010.02.001

Verwey, W. B., \& Dronkert, Y. (1996). Practicing a structured continuous key-pressing task: Motor chunking or rhythm consolidation? Journal of Motor Behavior, 28(1), 71-79.

Verwey, W. B., \& Eikelboom, T. (2003). Evidence for lasting sequence segmentation in the discrete sequence-production task. Journal of Motor Behavior, 35(2), 171-181.

Verwey, W. B., Lammens, R., \& van Honk, J. (2002). On the role of the SMA in the discrete sequence production task: A TMS study. Neuropsychologia, 40(8), 1268-1276.

Wambaugh, J. L., Nessler, C., Wright, S., \& Mauszycki, S. C. (2014). Sound production treatment: effects of blocked and random practice. American Journal of Speech-Language Pathology, 23(2), 225-245.

Willingham, D. B. (1998). A neuropsychological theory of motor skill learning. Psychological Review, 105(3), 558-584.

Wright, D. L. (1991). The role of intertask and intratask processing in acquisition and retention of motor-skills. Journal of Motor Behavior, 23(2), 139-145.

Wright, D. L., Li, Y., \& Coady, W. (1997). Cognitive processes related to contextual interference and observational learning: A replication of Blandin, Proteau and Alain (1994). Research Quarterly for Exercise \& Sport, 68(1), 106-109. 
Wright, D. L., Magnuson, C., \& Verwey, W. (2003). Developing response selection through high contextual interference training. Journal of Sport \& Exercise Psychology, 25, S140-S141.

Wright, D. L., Brueckner, S., Black, C. B., Magnuson, C., \& Immink, M. A. (2004). Long-term motor programming improvements occur via concatenation of movement sequences during random but not during blocked practice. Journal of Motor Behavior, 36(1), 39-50. doi: 10.3200/Jmbr.36.1.39-50

Wright, D. L., Magnuson, C. E., \& Black, C. B. (2005). Programming and reprogramming sequence timing following high and low contextual interference practice. Research Quarterly for Exercise and Sport, 76(3), 258-266.
Wulf, G., \& Lee, T. D. (1993). Contextual interference in movements of the same class - differential-effects on program and parameter learning. Journal of Motor Behavior, 25(4), 254-263.

Wymbs, N. F., \& Grafton, S. T. (2009). Neural substrates of practice structure that support future off-line learning. Journal of Neurophysiology, 102(4), 2462-2476. doi:10.1152/jn.00315.2009

Wymbs, N. F., Bassett, D. S., Mucha, P. J., Porter, M. A., \& Grafton, S. T. (2012). Differential recruitment of the sensorimotor putamen and frontoparietal cortex during motor chunking in humans. Neuron, 74(5), 936-946.

Young, D. E., Cohen, M. J., \& Husak, W. S. (1993). Contextual interference and motor skill acquisition - on the processes that influence retention. Human Movement Science, 12(5), 577-600. 